University of Wollongong

Research Online

Australian Institute for Innovative Materials -

Papers

Australian Institute for Innovative Materials

$1-1-2018$

Superhydrophobic Shape Memory Polymer Arrays with Switchable Isotropic/Anisotropic Wetting

Zhongjun Cheng

Harbin Institute of Technology, zcheng@uow.edu.au

Dongjie Zhang

Harbin Institute of Technology

Tong LV

Research Institute of Aerospace Special Materials and Processing Technology

Hua Lai

Harbin Institute of Technology

Enshuang Zhang

Research Institute of Aerospace Special Materials and Processing Technology

See next page for additional authors

Follow this and additional works at: https://ro.uow.edu.au/aiimpapers

Part of the Engineering Commons, and the Physical Sciences and Mathematics Commons

Research Online is the open access institutional repository for the University of Wollongong. For further information contact the UOW Library: research-pubs@uow.edu.au 


\title{
Superhydrophobic Shape Memory Polymer Arrays with Switchable Isotropic/ Anisotropic Wetting
}

\author{
Abstract \\ Smart surfaces with tunable wettability have aroused much attention in the past few years. However, to \\ obtain a surface that can reversibly transit between the lotus-leaf-like superhydrophobic isotropic and \\ rice-leaf-like superhydrophobic anisotropic wettings is still a challenge. This paper, by mimicking \\ microstructures on both lotus and rice leaves, reports such a surface that is prepared by creating micro/ \\ nanostructured arrays on the shape memory polymer. On the surface, the microstructure shapes can be \\ reversibly changed between the lotus-leaf-like random state and the rice-leaf-like 1D ordered state. \\ Accordingly, repeated switch between the superhydrophobic isotropic and anisotropic wettings can be \\ displayed. Research results indicate that the smart controllability is ascribed to the excellent shape \\ memory effect of the polymer, which endows the surface with special ability in memorizing different \\ microstructure shapes and wetting properties. Meanwhile, based on the smart wetting performances, the \\ surface is further used as a rewritable functional platform, on which various droplet transportation \\ programmes are designed and demonstrated. This work reports a superhydrophobic surface with \\ switchable isotropic/anisotropic wettings, which not only provides a novel functional material but also \\ opens a new avenue for application in controlled droplet transportation.

\section{Disciplines} \\ Engineering | Physical Sciences and Mathematics

\section{Publication Details} \\ Cheng, Z., Zhang, D., Lv, T., Lai, H., Zhang, E., Kang, H., Wang, Y., Liu, P., Liu, Y., Du, Y., Dou, S. Xue. \& Jiang, L. \\ (2018). Superhydrophobic Shape Memory Polymer Arrays with Switchable Isotropic/Anisotropic Wetting. \\ Advanced Functional Materials, 28 (7), 1705002-1-1705002-11.
}

\section{Authors}

Zhongjun Cheng, Dongjie Zhang, Tong Lv, Hua Lai, Enshuang Zhang, Hongjun Kang, Yongzhen Wang, Pengchang Liu, Yuyan Liu, Yi Du, Shi Xue Dou, and Lei Jiang 


\section{WILEY-VCH}

DOI: $10.1002 /$ ((please add manuscript number))

Article type: Full Paper

\section{Superhydrophobic Shape Memory Polymer Arrays with Switchable Isotropic/Anisotropic Wetting}

Zhongjun Cheng, * Dongjie Zhang, Tong Lv, Hua Lai, Enshuang Zhang, Hongjun Kang, Yongzhen Wang, Pengchang Liu, Yuyan Liu, * Yi Du, Shixue Dou and Lei Jiang

Dr. D. Zhang, Dr. H. Lai, Dr.H. Kang, Dr, Y. Wang, Dr. P. Liu, Prof. Y. Liu

MIIT Key Laboratory of Critical Materials Technology for New Energy Conversion and Storage, School of Chemistry and Chemical Engineering

Harbin Institute of Technology

Harbin 150001, P. R. China

E-mail: liuyy@hit.edu.cn

Prof. Z. Cheng

Natural Science Research Center

Academy of Fundamental and Interdisciplinary Sciences

Harbin Institute of Technology

Harbin 150090, P. R. China

E-mail: chengzhongjun@iccas.ac.cn

Dr. T. Lv, Dr. E. Zhang

Research Institute of Aerospace Special Materials and Processing Technology

Beijing 100174, P. R. China

Prof. L. Jiang

Beijing National Laboratory of Molecular Sciences (BNLMS)

Institute of Chemistry

Chinese Academy of Sciences

Beijing 100080, P. R. China

Prof. Y. Du, Prof. S. Dou

Institute for Superconducting and Electronic Materials

Australian Institute for Innovative Materials

University of Wollongong

Innovation Campus, North Wollongong, New South Wales 2500, Australia

Keywords: superhydrophobic, anisotropic wetting, shape memory polymer, tunable microstructure, reversible

Smart surfaces with tunable wettability have aroused much attention in the past few years. However, to obtain a surface that can reversibly transit between the lotus-leaf-like superhydrophobic isotropic and rice-leaf-like superhydrophobic anisotropic wettings is still a challenge. In this paper, by mimicking microstructures on both lotus and rice leaves, we report such a surface that was prepared by creating micro/nanostructured arrays on the shape memory polymer (SMP). On the surface, the microstructure shapes can be reversibly changed 


\section{WILEY-VCH}

between the lotus-leaf-like random state and the rice-leaf-like one dimensional ordered state. Accordingly, repeated switch between the superhydrophobic isotropic and anisotropic wettings can be displayed. Research results indicate that the smart controllability is ascribed to the excellent shape memory effect of the polymer, which endows the surface with special ability in memorizing different microstructure shapes and wetting properties. Meanwhile, based on the smart wetting performances, the surface was further used as a rewritable functional platform, on which various droplet transportation programmes were designed and demonstrated. This work reports a superhydrophobic surface with switchable isotropic/anisotropic wettings, which not only provides a novel functional material, but also opens a new avenue for application in controlled droplet transportation.

\section{Introduction}

In nature, many plants and insets have the superhydrophobicity. ${ }^{[1]}$ The most famous example is the lotus leaf, ${ }^{[1 \mathrm{c}, 1 \mathrm{~d}]}$ on which a water droplet has similar high contact angles (CAs) and low sliding angles (SAs) in all directions, representing the isotropic wetting. Rice leaf is also superhydrophobic, while it displays the anisotropic wetting, ${ }^{[1 \mathrm{c}]}$ a water droplet rolls more easily parallel to the leaf edge compared with the perpendicular direction. Research found that different wetting properties are ascribed to different surface microstructures: on the lotus leaves, the micro/nanopapillae are randomly distributed, while they are arranged in onedimensional order on the rice leaves. ${ }^{[1,2]}$ Inspired by these findings, lots of artificial superhydrophobic surfaces that imitating the random microstructures on louts leaves with isotropic wetting ${ }^{[3]}$ and quasi-1D microstructures on rice leaves with anisotropic wetting, ${ }^{[4]}$ respectively, have been prepared. However, all these surfaces inspired by single natural microstructure can display only a constant isotropic or anisotropic wetting performance. Recently, multi-biomimetic strategy is creating fresh ideas in designing artificial materials since it can advance new functions beyond the natural biology. ${ }^{[5]}$ For example, learned from 


\section{WILEY-VCH}

lotus leaf and pitcher plants, Huang et al., reported a magnetically responsive micropillars that can not only switch between lotus leaf's superhydrophobic mode and pitcher plant's slippery mode, but also display programmable fog harvesting and transportation ability. ${ }^{[5]}$ Herein, based on similar multi-biomimetic strategy and learned from microstructures on both lotus leaf and rice leaf, we designed a surface with dynamically tunable microstructures that can change between the lotus-leaf-like random shape and rice-leaf-like quasi-1D groove shape. It was found that the surface can not only display lotus-leaf-like superhydrophobic isotropic and rice-leaf-like anisotropic wettings, but also can realize the smart transition between the two states. More importantly, based on the smart wetting performance, the surface can be used as a rewritable microstructure-dependent platform, on which different droplet transportation programmes can be achieved. To the best of our knowledge, although lots of smart superhydrophobic surfaces with switchable wettability have been reported, ${ }^{[3 a, 6-8]}$ none of them can demonstrate the dynamically switching between the lotus-leaf-like superhydrophobic isotropic and rice-leaf-like superhydrophobic anisotropic wettings.

In the past few years, researches about surface anisotropic wetting property have aroused much attention, ${ }^{[9-12]}$ especially for those superhydrophobic surfaces, ${ }^{[4 c-4 f,}{ }^{13-15]}$ such as hierarchical microgrooves structured polydimethylsiloxane (PDMS) ${ }^{[4 c]}$ periodic isosceles triangle micro-arrays, ${ }^{[4 \mathrm{~d}]}$ micro-wrinkled wavy surfaces, ${ }^{[4 \mathrm{f}]}$ etc. which integrated both superhydrophobicity and anisotropic wetting have demonstrated excellent controllability for water droplets and been applied in many applications, for example, self-propelled leaping of droplet, liquid transportation/separation, and microfluidic devices, etc. It is worthy of noting that the anisotropic characteristics including anisotropic degree and anisotropic direction are all constant on almost all these surfaces since the chemical/geometrical structures are fixed, which significantly limits their applications in more complex smart device. A superhydrophobic surface with tunable wettability between the isotropic and anisotropic wetting states would be promising to overcome this imperfection and provide many novel 


\section{WILEY-VCH}

applications, for example, as the rewritable platform for designing different anisotropic wetting performances, but until now, it is still a challenge. As mentioned above, different microstructure arrangements for lotus leaf and rice leaf endow them with isotropic and anisotropic wettings, respectively. ${ }^{[1 \mathrm{c}]}$ Dynamically controlling surface microstructure is believed to be an effective way to realize the switch between the isotropic and anisotropic wetting. Recently, some efforts have been expended on the elastic PDMS substrates to attempt to realize this goal by repeatedly stretching/relaxing or controlling one-direction curvature of the substrates. ${ }^{[16]}$ However, only static switch between isotropic and anisotropic wettings can be achieved since these surfaces display high adhesion to water droplet (droplet cannot roll, which is contrary to the phenomena on the lotus leaf and rice leaf). Meanwhile, such elastic surfaces cannot keep the deformed microstructures and related wetting performances without the external actions (for instance, mechanical strain), thus limiting their applications in some fields where external actions are unsuitable. In this regard, to advance new strategies and novel materials for realizing reversible transition between the lotus-leaflike superhydrophobic isotropic wetting and rice-leaf-like superhydrophobic anisotropic wetting are still urgently needed.

In this work, inspired by the special wetting performances and microstructure shapes on the lotus leaf and rice leaf, we report a surface that can transit reversibly between the superhydrophobic isotropic and anisotropic wettings through dynamically controlling the microstructure shape to imitate lotus-leaf-like structure and rice-leaf-like structure, respectively (Figure 1a). The surface was prepared by creating micro/nanostructured pillars on the shape memory polymer (SMP) material through polymerizing the diglycidyl ether of bisphenol A type epoxy resin (DGEBA), n-octylamine (OA), and m-xylylenediamine (MXDA) on a micro/nanostructured template (Figure S1-S3 in the supporting information). The good shape memory effect of the polymer allows the surface to repeatedly memorize and display different microstructure shapes, and ultimately resulted in the smart transition 


\section{WILEY-VCH}

between the two wetting states. Furthermore, based on the smart transition ability, we showed that the surface can be used as the rewritable platform, on the surface, different water transportation programmes can be achieved by designing different grooves structures, which successfully overcome the current problem that one surface often can display only a fixed anisotropic wetting performance. As we know, although a lot of studies have been focused on SMP materials due to their special shape memory ability and related important applications including in biomedical devices, smart adhesives, and optical chips, ${ }^{[17-18]}$ studies of the superhydrophobic SMP are still rare and have only been reported in very recently for selfcleaning, water shedding, and so on. ${ }^{[19]}$ This work reports a superhydrophobic surface with switchable isotropic/anisotropic wettings, which not only provides a novel functional material, but also opens a new avenue for application in controlled droplet transportation.

\section{Results and discussion}

\subsection{Morphology and wetting switching}

Figure 1b-d displays the scanning electron microscopy (SEM) images of the as-prepared surface at different amplified scales. It can be seen that regular pillars with diameter, spacing and height of about $10 \mu \mathrm{m}, 10 \mu \mathrm{m}$, and $30 \mu \mathrm{m}$, respectively, covering the surface uniformly (Figure 1b, 1c). Amplified image demonstrates that nanopits with width of about $520 \mathrm{~nm}$ dispersing on the pillar tips (Figure 1d). These results indicate that the as-prepared surface has similar micro/nanostructures with those on the lotus leaf. ${ }^{[1 c]}$ On such a surface with uniform micro/nanostructured pillars, a water droplet $(4 \mu \mathrm{L})$ has the same CAs $\left(151 \pm 1.5^{\circ}\right)$ and SAs $\left(24 \pm 1.2^{\circ}\right)$ measured in arbitrary two vertical directions $\mathrm{A}$ and $\mathrm{B}$, respectively (insets in Figure 1b, Figure 1h), indicating that the surface has the superhydrophobic isotropy in both static and dynamic wettability (herein, results measured in directions A and B just represent some examples, in fact, the CA and SA are similar in random directions on the surface). To obtain the rice-leaf-like microstructures, silicon templates with microgroove structure was used to press the surface (at $120^{\circ} \mathrm{C}$ that higher than the glass transition temperature $\left(\mathrm{T}_{\mathrm{g}}\right.$ ) of the 


\section{WILEY-VCH}

polymer, Figure S4-S6 in supporting information). Figure 1e shows the SEM image of the surface that after pressing by a template with groove width $\left(\mathrm{W}_{\mathrm{g}}\right)$ of about $260 \mu \mathrm{m}$. One can clearly see that new microgroove structure can be formed on the surface. From magnified images, it can be seen that some pillars remain constant (those unpressed pillars), both the morphology and the size are similar with the original states (Figure 1f), while those pressed pillars collapse and show uniform deformation and orientation (Figure 1g). Like the rice leaf, such a microgroove structured surface displays superhydrophobic anisotropic wetting. As shown in Figure 1i, in direction $\mathrm{B}\left(\mathrm{CA}=151 \pm 2^{\circ}, \mathrm{SA}=29 \pm 1.5^{\circ}\right.$, inset in Figure 1e, measured perpendicular to the grooves), both $\mathrm{CA}$ and SA are larger than those in direction $\mathrm{A}$ $\left(\mathrm{CA}=142 \pm 1.5^{\circ}, \mathrm{SA}=20 \pm 1.3^{\circ}\right.$, measured parallel to the grooves, inset in Figure $\left.1 \mathrm{e}\right)$. Further heating the surface at $120^{\circ} \mathrm{C}$ for about $45 \mathrm{~s}$, one can observe that both the surface microstructure and the wetting performances return to the initial state (Figure S7 in supporting information), and such transition can be repeated several cycles without any deterioration of the responsivity (Figure 1j, take the dynamic anisotropy as an example, the static wetting changes see supporting information Figure S8). These results indicate that reversible transition between the superhydrophobic isotropic and anisotropic wetting states can be realized by controlling surface microstructure shape between the lotus-leaf-like structure and the rice-leaf-like structure repeatedly. Herein, what needs to be stressed is that the surface chemical composition has no apparent change during the variation of surface microstructure (Table S1 in supporting information), means that the microstructure variation mainly induces the smart wetting performance.

As mentioned above, it is the surface microstructure variation that leads to the switch of the surface wetting performance, to have a better insight into the behaviors of surface microstructure, its variation was further in-situ investigated by a confocal microscopy. Figure $2 \mathrm{a}$ and $2 \mathrm{~b}$ show the $3 \mathrm{D}$ confocal microscopy image and corresponding profile picture of the as-prepared surface. It can be seen that both the morphology and the size of the pillars are in 


\section{WILEY-VCH}

good agreement with the results derived from the SEM (Figure 1b-d). Atomic force microscopy (AFM) image further confirms the presence of nanostructure on the top part of pillar and the roughness is about $24.5 \mathrm{~nm}$ (Figure 2c). After pressing by the template, as shown in Figure $2 \mathrm{~d}$ (the $\mathrm{W}_{\mathrm{g}}$ of template is about $100 \mu \mathrm{m}$ ), some pillars collapse while others remain unchanged. From the corresponding profile picture (Figure 2e), it can be seen that the average pillar height decreases to about $9.2 \mu \mathrm{m}$ for those collapsed pillars, and remains constant at about $30 \mu \mathrm{m}$ for those unchanged pillars. Under heating at $100^{\circ} \mathrm{C}$, one can observe that the average heights for those collapsed pillars increase while for those unchanged pillars remain constant (Figure 2f). When the time is increased to about $110 \mathrm{~s}$, both the morphology (Figure 2g) and the size (Figure 2h) of the pillars would restore to the original state. In addition, we also find that the recovery velocity of the collapsed pillars is dependent on the heating temperature, and the needed time for total recovery is decreased with increasing the temperature (Figure S9 in supporting information). Moreover, due to its intrinsic 3D net crosslinking structures of epoxy SMP, the material has remarkable fatigue durability (Figure S10 in supporting information), the microstructure can be recovered even after pressing several times (Figure 2i). Accordingly, as shown in Figure $1 \mathrm{j}$, surface wettability can be repeatedly switched between the superhydrophobic isotropic and anisotropic wetting states. Moreover, the smart transition can still be observed even after one month, demonstrating a good stability of our surface.

To obtain a surface has the most remarkable superhydrophobic anisotropic wetting performance, arrays of pillars with different spacing, height, and $\mathrm{W}_{\mathrm{g}}$ were investigated. Figure 3a shows the results of CAs and SAs on $30 \mu \mathrm{m}$ height pillars with spacing of $5 \mu \mathrm{m}, 10 \mu \mathrm{m}, 20$ $\mu \mathrm{m}$, and $30 \mu \mathrm{m}$, respectively (before pressing and without groove structures). One can observe that water droplets can roll on all these surfaces with high CAs (higher than $150^{\circ}$ ). Herein, only arrays with spacing of $10 \mu \mathrm{m}$ was chosen to introduce groove structure since other surfaces have unrecoverable microstructure or would lose the superhydrophobicity after 


\section{WILEY-VCH}

pressing by the template (Figure S11-S13 in supporting information). Figure 3b-f display the relationship between the CAs/SAs and the $\mathrm{W}_{\mathrm{g}}$ on pillars with different heights $(10 \mu \mathrm{m}, 20 \mu \mathrm{m}$, and $30 \mu \mathrm{m}$, more information sees Figure S14 in supporting information). When the pillar height is too low $(10 \mu \mathrm{m})$, only static anisotropic wetting can be observed because the surface superhydrophobicity would disappear after introduction of microgroove structure (droplets be pinned in directions both parallel and perpendicular to the grooves, Figure $3 \mathrm{~b}, \mathrm{SA}=90^{\circ}$ means that droplet is pinned on the surface). When the pillar height is increased to $20 \mu \mathrm{m}$, the surfaces can remain the superhydrophobicity when the $\mathrm{W}_{\mathrm{g}} \leq 220 \mu \mathrm{m}$ (Figure 3c). However, on these pillars, the difference values for $\mathrm{CA}(\Delta \mathrm{CA})$ and $\mathrm{SA}(\Delta \mathrm{SA})$ between two investigation directions (perpendicular and parallel to the grooves) are too small, means that the wetting anisotropy is inconspicuous (Figure 3c, 3d). Further increasing the pillar height to $30 \mu \mathrm{m}$, we can find that surface can keep the superhydrophobicity even when then $\mathrm{W}_{\mathrm{g}}$ is as large as 260 $\mu \mathrm{m}$ (Figure 3e, when $\mathrm{W}_{\mathrm{g}}>260 \mu \mathrm{m}$, surface would lose the superhydrophobicity in static wettability, CAs in both directions parallel and perpendicular to grooves would less than $150^{\circ}$ ). More importantly, the increase of $\mathrm{W}_{\mathrm{g}}$ can effectively increase the wetting anisotropy. On such a surface (pillar height of $30 \mu \mathrm{m}, \mathrm{W}_{\mathrm{g}}=260 \mu \mathrm{m}$ ), the $\Delta \mathrm{CA}$ and $\Delta \mathrm{SA}$ are $9^{\circ}$ and $9^{\circ}$, respectively (Figure 3e, 3f), which is similar with the wetting performance on the rice leaf. ${ }^{[4 c, 13 e]}$ From the above comparison and analysis, two rules can be deduced. Firstly, high pillars can help the surface remain the superhydrophobicity after introduction of microgrooves; secondly, large $\mathrm{W}_{\mathrm{g}}$ is in favor of the increase of wetting anisotropy (more discussion see supporting information). ${ }^{[4 c]}$ In this work, the surface composed of pillars with height of $30 \mu \mathrm{m}$ and $\mathrm{W}_{\mathrm{g}}$ of $260 \mu \mathrm{m}$ has the best superhydrophobic anisotropy.

\subsection{Mechanism of the smart wettability}

From the above, it can be seen that good controllability of surface microstructure (transition between lotus-leaf-like and rice-leaf-like structure shapes) and suitable microstructure sizes (including pillar height and $\mathrm{W}_{\mathrm{g}}$ ) are important for the smart wetting 


\section{WILEY-VCH}

performances, which can be explained as follows. The tunable microstructure can be ascribed to the good shape memory effect of the polymer, which can memorize and display various microstructure shapes (Figure S15 in supporting information). ${ }^{[20]}$ Briefly, the lotus-leaf-like micro/nanostructured pillars on the as-prepared surface are the permanent shape (Figure 4a), the molecular chains adopt conformation with the highest entropy and in the thermodynamically stable state (Figure $4 \mathrm{~g}$ ). ${ }^{[21]}$ When the surface is heated above the $\mathrm{T}_{\mathrm{g}}$, the molecular chains mobility is activated, and the polymer becomes soft (Figure 4b). In this situation, when a template with groove structure is used to press the surface, those pillars be pressed would collapse while others keep the pristine state (Figure 4c). It is worthy of noting that during this process, the crosslinking network formed during the polymerization between DGEBA, OA, and MXDA can effectively prohibit the long-rang chain slippage, which can cause the change of the chain conformation and realize the entropy trapping. ${ }^{[21]}$ As a result, those collapsed pillars changes into a low entropy state (Figure 4h), and such a state can be kept after further cooling the surface under the external force below the $T_{g}$ because of the freeze of the molecular chain segments (the undeformed pillars would return to the initial thermodynamically stable state), finally forming the groove structure on the surface (Figure 4d). Upon reheating the surface above the $T_{g}$ without external load, all the molecular chains mobility is re-activated (Figure 4e). For those undeformed pillars, the molecular chain configuration keeps constant, while for those deformed pillars, the thermodynamically favorable tendency for increasing entropy allows the molecular configuration to change back to its permanent configuration, and collapsed pillars would be recovered (Figure 4f). After further cooling to the room temperature, the surface microstructure would restore to the initial lotus-leaf-like shape. Therefore, as shown in Figure 2i, surface microstructure can be repeatedly controlled between the lotus-leaf-like structure and rice-leaf-like structure through cycled pressing/recovering processes. 


\section{WILEY-VCH}

Based on the above explanation, it would be easy to understand the variation of surface microstructure between the lotus-leaf-like and the rice-leaf-like structure shapes. It is well known that different microstructures can lead to different wetting performances. ${ }^{[1 \mathrm{c}]}$ On the original surface, a regular arrangement of the micro/nanostructured pillars can be observed under the water droplet (Figure 4i, the micro/nanostructure can help to trap air and lead to the surface superhydrophobicity ${ }^{[22]}$, more discussion about the superhydrophobicity see supporting information). When a water droplet is wetting and rolling along two arbitrary vertical directions on the surface (Figure $4 \mathrm{j}$ and 41 ), the energy barriers needed to be overcame are similar (Figure 4k and 4m, the similar energy barrier is due to the same widths of gaps that the droplet needs to jump across), ${ }^{[1 \mathrm{c}, 2,23]}$ and the length/continuity of three-phase contact line (TCL) are also alike (Figure $4 \mathrm{i})^{[12 \mathrm{~b}]}$. Therefore, as shown in Figure 1h, the water droplet has the same CA and SA in the two vertical directions and the surface shows the lotus-leaf-like superhydrophobic isotropic wetting. Herein, what needs to be stressed is that two vertical directions presented in Figure 4i just represent some examples. In fact, the surface shows the superhydrophobic isotropic wettability in random directions since the energy barriers are approximate between each other in all directions (Figure S17 and related discussion in supporting information). After introduction of the microgrooves, the grooves can squeeze/pin and stretch the droplet in directions viewed parallel and perpendicular to the grooves, respectively. ${ }^{[11 \mathrm{~d}]}$ As a result, the CA measured perpendicular to the grooves is larger than that measured parallel to grooves (Figure 1i). Meanwhile, it can be found that different from the direction A (measured direction parallel to the grooves, Figure 4n), much larger gaps are present in direction B (measured direction perpendicular to the grooves, Figure 4n). As reported, ${ }^{[23]}$ in composite Cassie wetting state, larger gaps can offer larger energy barrier. When a droplet is rolling in direction A (along the grooves), it only needs to jump across the small valleys between pillars (Figure 4o) with low energy barrier (Figure 4p), and the TCL is relative continuous (Figure 4n). When the droplet is rolling in direction B (perpendicular to 


\section{WILEY-VCH}

the grooves), compared with direction A, in addition to the small valleys between pillars, it also needs to jump across big groove gaps with higher energy barrier (Figure 4q and 4r), and the TCL would be more discontinuous (Figure 4n). Therefore, similar as that on the rice leaf and as shown in Figure 1i, the SA is higher in direction perpendicular to grooves than that parallel to grooves because the TCL is more discontinuous in the perpendicular direction due to higher wetting/dewetting energy barrier. ${ }^{[4 f, 12 b]}$ Noticeably, to obtain remarkable superhydrophobic anisotropic wetting, suitable structure sizes including pillar height and $\mathrm{W}_{\mathrm{g}}$ are also important. On surfaces with short pillars, after introduction of microgroove, such shorts pillars cannot withstand the droplet and impede the water wetting the gaps due to the decrease of the upward Laplace force (Figure S18a in supporting information). ${ }^{[12 b, 24]}$ Therefore, as shown in Figure 3b, surface with pillar height $10 \mu \mathrm{m}$ would lose the superhydrophobicity after introducing the groove structure. Furthermore, $\mathrm{W}_{\mathrm{g}}$ is also significant, as the $\mathrm{W}_{\mathrm{g}}$ is too small, it cannot provide effect energy barrier to cause the anisotropy (Figure $\mathrm{S} 18 \mathrm{~b}$ in supporting information), while when the $\mathrm{W}_{\mathrm{g}}$ is too large, the pillar height become insufficient to prevent water from wetting the gaps (Figure S18c in supporting information). Therefore, as shown in Figure 3c-f, the degree of anisotropy is increased as the $\mathrm{W}_{\mathrm{g}}$ is increased, while finally lose the superhydrophobicity as the $\mathrm{W}_{\mathrm{g}}$ is too large (more discussion see supporting information). From the above, it can be seen that the absence or presence of groove structures endow the pillar-structured surface with superhydrophobic isotropic and anisotropic wettings, respectively. As explained in the above paragraph, the groove structures can be introduced and removed repeatedly due to the excellent shape memory effect of the polymer, therefore, as shown in Figure 1j, cycled switching between the superhydrophobic isotropy and anisotropy can be realized.

\subsection{Application of the surface as a rewritable platform for droplet transportation}

The surface with such a special switchable ability in superhydrophobic isotropic and anisotropic wettings can be potentially used in many applications, such as in microdroplet- 


\section{WILEY-VCH}

related functional chip. ${ }^{[25]}$ Recently, such superhydrophobic chips have aroused much attention due to their wide applications in microreaction and cell adhesion/transfection. During these applications, realization the controllable droplet transportation is very important, and design anisotropic wetting on the surface is a common way to obtain such controllable transportation chips ${ }^{[26]}$ However, almost all present transportation chips, especially those with microstructure-dependent anisotropic wettability, can demonstrate only a single transportation programme and often are difficult be redesigned since the surface microstructures often are static. It is worthy of noting that our surface can be used as a rewritable platform, on which, various droplet transportation programmes can be achieved. As a demonstration, we designed groove structures on the bottom half of our surface through pressing with the groove structured template, and then investigated the water droplets transportation properties as a water droplet was rolling from top to bottom on the surface (the surface is tilted at about $25^{\circ}$ ). As shown in Figure 5, groove structures $\left(\mathrm{W}_{\mathrm{g}}=260 \mu \mathrm{m}\right)$ with various directions can be created on our surface through repeatedly pressing/recovering processes. When the groove structure is designed in the horizontal direction (Figure 5a), a water droplet (about $10 \mu \mathrm{L}$, dyed with methylene blue for clearly observation) can roll in the region without groove structure (this regions shows superhydrophobic isotropy) and be pinned as it reaches the region with groove structures (this regions shows superhydrophobic anisotropy). (Figure $5 \mathrm{a}_{1}-\mathrm{a}_{4}$ ). As the groove direction is changed into vertical direction (Figure 5b), lean to the left (Figure 5c), and lean to the right (Figure 5d), respectively, it can be seen that when the droplet gets to the regions with groove structures, transportation directions can be easily changed along the groove directions (vertical: Figure $5 b_{1}-b_{4}$; lean to the left: Figure $5 c_{1}-c_{4}$; leant to the right: Figure $5 d_{1}-d 4$ ), demonstrating excellent controllability for droplet transportation. It is well known that surface adhesion is the key factor that determines the dynamical property of a droplet on the surface,${ }^{[27]}$ and different sliding properties as described above can be ascribed to the effect of the adhesion anisotropy. ${ }^{[2,11 a, 12 c]}$ As already reported, groove structured surfaces have such 


\section{WILEY-VCH}

adhesion anisotropy, they display high/low water adhesions in directions that perpendicular/parallel to the grooves, respectively. ${ }^{[2]}$ The adhesion difference in different directions can effectively prevent the water droplet from rolling in the high adhesive direction and restrict it to slide along the low adhesive direction. ${ }^{[26 a]}$ Therefore, as shown in Figure 5, the sliding water droplets could be pinned or turned along the groove direction as they reached the groove structured regions. From the above, it can be concluded that based on our surface, different droplet transportation plans can be achieved due to the particular shape memory microstructure and switchable superhydrophobic isotropic and anisotropic wettability. Furthermore, it needs to be stressed that droplet transportation just represents one function, it is believed that the surface with such smart ability can be easily extended to other microstructure-dependent functional application, such as optics, friction, and energy transfer.

\section{Conclusions}

In conclusion, a smart superhydrophobic surface with shape memory micro/nanostructure is reported, on which the surface microstructure can be controlled reversibly between the lotus-leaf-like structure and the rice-leaf-like structure, accordingly, the wetting performances can be switched reversibly between the superhydrophobic isotropic and anisotropic states. The smart controllability is ascribed to the good shape memory effect at micro/nanoscale of the polymer, which can result in different microstructure shapes and solid/liquid wettings. Furthermore, based on the special switchable ability, the novel application of the surface as a rewritable platform for controllable droplet transportation was also demonstrated. This work advances a strategy to realize the dynamic control of the surface microstructure based on the shape memory effect of SMP, and firstly reports a surface that can switch reversibly between the lotus-leaf-like superhydrophobic isotropic wetting state and the rice-leaf-like superhydrophobic anisotropic wetting state. Given the smart wetting performance on the surface, it is believed to be potentially used in many other applications, such as bioseparation, and the microfluidic device. Meanwhile, the concept can easy be 


\section{WILEY-VCH}

extended to other SMPs with different external stimulus, for instance, light, magnetic, and electric.

\section{Experimental Section}

Fabrication of the surface: The hierarchical structured superhydrophobic SMP surfaces were prepared by the replica-molding method. The hierarchical structured silicon template was firstly prepared by combing the photolithography and chemical etching process. After that, PDMS mold was obtained by replicating the rough silicon substrate. Finally, superhydrophobic SMP surface was prepared through the replication process on the PDMS mold. In detail, Contact lithographic masks were constructed by Microelectronics R\&D Center, the Chinese Academy of Sciences. A KARL SUSS MA6 (Germany) instrument was used to transfer the patterns of masks onto silicon wafers by a photolithographic method. A deep-etching process was completed using an STSICP ASE (UK) instrument. Thus rough silicon wafers were prepared on which geometrical structures of patterned square pillars were introduced. To obtain the hierarchical micro/nanostructure, a metal-assisted chemical etching process was used to introduce nanostructure onto the pillars. Pillar structured silicon substrate was firstly treated with piranha solution (mixture of $\mathrm{H}_{2} \mathrm{SO}_{4}(98 \%)$ and $\mathrm{H}_{2} \mathrm{O}_{2}(30 \%)$ at $\mathrm{V} / \mathrm{V}=3: 1)$ at $80^{\circ} \mathrm{C}$ for $1 \mathrm{~h}$, and then coated by the $\mathrm{Ti}(2 \mathrm{~nm})$ and $\mathrm{Au}(5 \mathrm{~nm})$ layers in sequence. After that, the substrate was immersed into the etching solution (1:1 $\mathrm{HF}(40 \%)$ and $\mathrm{H}_{2} \mathrm{O}_{2}$ $(30 \%))$ for about $30 \mathrm{~s}$, and then treated with aquaregia (1:3 volume mixture of $69 \% \mathrm{HNO}_{3}$ and $37 \% \mathrm{HCl}$ ) for $1 \mathrm{~min}$ to remove the remainder Au. Finally, the substrate was taken out, washed with abundant pure water and dried with $\mathrm{N}_{2}$.

The hierarchical structured silicon substrate was firstly modified with FAS (Heptadecafluorodecyltrimethoxysilane, shin-Etsu chemical Co., Ltd., Japan), which was conducted in an evacuated desiccator for one night under the vacuum condition using $40 \mu \mathrm{L}$ of FAS. ${ }^{[18 \mathrm{~d}]}$ After that, the PDMS precursors were poured over the FAS modified structured silicon substrate. After degassing for about 15 min under vacuum, the prepolymer mixture 


\section{WILEY-VCH}

was cured at $65^{\circ} \mathrm{C}$ for $4 \mathrm{~h}$ and the PDMS mold can be obtained after peeling off carefully from the silicon substrate.

The prepolymer of SMP was mixed by bisphenol A diglycidyl ether (Bluestar Chemical New Materials Co), n-octylamine (J\&K Scientific) and m-xylylenediamine (Changsha institute of chemical industry) in a molar ratio of 8:2:3. After degassing for $10 \mathrm{~min}$, the SMP prepolymer mixture was poured onto a PDMS mould and baked at $60^{\circ} \mathrm{C}(2 \mathrm{~h})$ and $100^{\circ} \mathrm{C}(1 \mathrm{~h})$ in sequence. Finally, the superhydrophobic SMP with hierarchical micro/nanostructures was achieved after carefully peeling.

Controlling the surface microstructure: To obtain the rice-leaf-like structure on the surface, a template with groove structure (Figure S4) was used to press the surface. Detailed process as shown in Figure S5. the surface was firstly heated at $120^{\circ} \mathrm{C}$ for about $5 \mathrm{~min}$, followed by pressing using a pre-cleaned microgroove structured silicon template under a certain pressure (Figure S5a-b). Noticeably, to assure that those pillar contact with the concave section of template have no deformation, the used template with depth of groove $(50 \mu \mathrm{m})$ must be larger than the height of pillar $(30 \mu \mathrm{m})$. After pressing, the surface was cooled to temperature under the pressure (Figure S5b-c), and then the microgroove structures can be formed on the surface after demolding (Figure S5d). For restoration to the original state, the deformed surface was reheated at $120^{\circ} \mathrm{C}$ for a certain time in an oven (Figure S5e), and finally, the microstructures would be restored during the process. After cooling to the room temperature, the surface microstructure would restore to the initial state.

Characterization: The surface morphologies were measured on an atomic force microscope (AFM, Bruker, Dimension Icon) and a field-emission SEM apparatus (HITACHI, SU8010). The 3D morphology and corresponding profile curves were recorded on a confocal microscope (Olympus, OLS3000). The water CAs and SAs were measured by a contact angle measure meter (JC 2000D5, Shanghai Zhongchen Digital Technology Apparatus Co., Ltd). 


\section{WILEY-VCH}

The final values were obtained by averaging five different values from different positions on the same surface. The Dynamic Mechanical Analysis (DMA) measurements data were obtained with a Mettler SDTA861e analyzer. The data contains storage modulus and loss tangent $(\tan \delta)$ of the specimens. Tensile tests were carried out using a tensile tester (Instron 5965) to evaluate the mechanical strength of materials. Shape memory properties were performed with a TA Q800 dynamic mechanical analyzer (USA).

\section{Supporting Information}

Supporting Information is available from the Wiley Online Library or from the author.

\section{Acknowledgements}

This work is supported by the National Natural Science Foundation of China (NSFC grant nos. 21674030, and 51573035).

References

Received: ((will be filled in by the editorial staff))

Revised: ((will be filled in by the editorial staff)) Published online: ((will be filled in by the editorial staff))

[1] a) M. Liu, S. Wang, L. Jiang, Nat. Rev. Mater. 2017, 2, 17036; b) D. Vella, Science 2015, 349, 472; c) L. Feng, S. Li, Y. Li, H. Li, L. Zhang, J. Zhai, Y. Song, B. Liu, L. Jiang, D. B. Zhu, Adv. Mater. 2002, 14, 1857; d) W. Barthlott, C. Neinhuis, Planta 1997, 202, 1.

[2] D. Xia, L. M. Johnson, G. P. López, Adv. Mater. 2012, 24, 1287.

[3] a) S. T. Wang, K. S. Liu, X. Yao, L. Jiang, Chem. Rev. 2015, 115, 8230; b) T. Jiang, Z. Guo, W, Liu, J. Mater. Chem. A, 2015, 3, 1811; c) P. Ragesh, V. A. Ganesh, S. V. Naira, A. S. Nair, J. Mater. Chem. A 2014, 2, 14773; d) T. Darmanin, F. Guittard, J. Mater. Chem. A 2014, 2, 16319 .

[4] a) G. D. Bixler, B. Bhushan, Nanoscale. 2013, 5, 7685; b) G. D. Bixler, B. Bhushan, Nanoscale. 2014, 6, 76; c) D. Wu, J. Wang, S. Wu, Q. Chen, S. Zhao, H. Zhang, H. Sun, L. Jiang, Adv. Funct. Mater. 2011, 21, 2927; d) J. Liu, H. Guo, B. Zhang, S. Qiao, M. Shao, X. Zhang, X. Feng, Q. Li, Y. Song, L. Jiang, J. Wang, Angew. Chem. Int. Ed. 2016, 55, 4265; e) S. M. Kang, C. Lee, H. N. Kim, B. J. Lee, J. E. Lee, M. K. Kwak, K. Suh, Adv. Mater. 2013, 


\section{WILEY-VCH}

25, 5756; f) S. G. Lee, H. S. Lim, D. Y. Lee, D. Kwak, K. Cho, Adv. Funct. Mater. 2013, 23, 547; g) J. Long, P. Fan, D. Jiang, J. Han, Y. Lin, M. Cai, H. Zhang, M. Zhong, Adv. Mater. Interface 2016, 1600641.

[5] a) Y. Huang, B. B. Stogin, N. Sun, J. Wang, S. Yang, T. Wong, Adv. Mater. 2017, 29, 1604641; b) M. Cao, X. Jin, Y. Peng, C. Yu, K. Li, K. Liu, L. Jiang, Adv. Mater. 2017, 29, 1606869.

[6] a) Y. Lai, J. Huang, Z. Cui, M. Ge, K. Zhang, Z. Chen, L. Chi, Small 2016, 12, 2203; b) X. Huang, Y. Sun, S. Soh, Adv. Mater. 2015, 27, 4062; c) J. Lahann, S. Mitragotri, T. N. Tran, H. Kaido, J. Sundaram, I. S. Choi, S. Hoffer, G. A. Somorjai, R. Langer, Science 2003, $299,371$.

[7] a) A. Grigoryev, I. Tokarev, K. G. Kornev, I. Luzinov, S. Minko, J. Am. Chem. Soc. 2012, 134, 12916; b) X. Yao, Y. Hu, A. Grinthal, T. S. Wong, L. Mahadevan, J. Aizenberg, Nat. Mater. 2013, 12, 529; c) Y. Y. Zhu, D. S. Antao, R. Xiao, E. N. Wang, Adv. Mater. 2014, 26, 6442; d) S. G. Lee, D. Y. Lee, H. S. Lim, D. H. Lee, S. Lee, K. Cho, Adv. Mater. 2010, 22, 5013.

[8] a) D. Wu, S. Z. Wu, Q. D. Chen, Y. L. Zhang, J. Yao, X. Yao, L. G. Niu, J. N. Wang, L. Jiang, H. B. Sun, Adv. Mater. 2011, 23, 545; b) X. Yu, Z. Q. Wang, Y. G. Jiang, F. Shi, X. Zhang, Adv. Mater. 2005, 17, 1289; c) H. S. Lim, D. Kwak, D. Y. Lee, S. G. Lee, K. Cho, J. Am. Chem. Soc. 2007, 129, 4128.

[9] a) N. A. Malvadkar, M. J. Hancock, K. Sekeroglu, W. J. Dressick, M. C. Demirel, Nat. Mater. 2010, 9, 1023; b) M. Yilmaz, H. B. Kuloglu, H. Erdogan, S. S. Cetin, M. S. Yavuz, G. O. Ince, G. Demirel, Adv. Mater. Interfaces 2015, 2, 1500226; c) C. Huang, M. Lai, W. Liu, Z. Wei, Adv. Funct. Mater. 2015, 25, 2670.

[10] a) P. Zhang, H. Liu, J. Meng, G. Yang, X. Liu, S. Wang, L. Jiang, Adv. Mater. 2014, 26, 3131; b) V. Jokinen, M. Leinikka, S. Franssila, Adv. Mater. 2009, 21, 4835; c) T. Guo, P. Che, L. Heng, L. Fan, L. Jiang, Adv. Mater. 2016, 28, 6999; d) M. Zhu, Y. Wang, S. Zhu, L. 


\section{WILEY-VCH}

Xu, C. Jia, J. Dai, J. Song, Y. Yao, Y. Wang, Y. Li, D. Henderson, W. Luo, H. Li, M. L. Minus, T. Li, L. Hu, Adv. Mater. 2017, 29, 1606284.

[11] a) A. Nakajima, NPG Asia Mater. 2011, 3, 49; b) D. Xia, X. He, Y. Jiang, G. P. López,

S. R. J. Brueck, Langmuir 2010, 26, 2700; c) H. Kusumaatmaja, R. J. Vrancken, C. W. M. Bastiaansen, J. M. Yeomans, Langmuir 2008, 24, 7299; d) Y. Chen, B. He, J. Lee, N. A. Patankar, J. Colloid. Interf. Sci. 2005, 281, 458.

[12] a) L. Kubus, H. Erdogan, E. Piskin, G. Demirel, Soft Matter. 2012, 8, 11704; b) Z. Yoshimitsu, A. Nakajima, T. Watanabe, K. Hashimoto, Langmuir 2002, 18, 5818; c) M. Morita, T. Koga, H. Otsuka, A. Takahara, Langmuir 2005, 21, 911; d) S. Wang, N. Yu, T. Wang, P. Ge, S. Ye, P. Xue, W. Liu, H. Shen, J. Zhang, B. Yang, ACS Appl. Mater. Interfaces 2016, 8, 13094.

[13] a) W. Li, G. Fang, Y. Li, G. Qiao, J. Phys. Chem. B 2008, 112, 7234; b) J. Zhou, A. V. Belyaev, F. Schmid, O. I. Vinogradova, J. Chem. Phys. 2012, 136, 194706; c) H. Kusumaatmaja, J. M. Yeomans, Soft Matter. 2009, 5, 2704; d) F. Chen, D. Zhang, Q. Yang, X. Wang, B. Dai, X. Li, X. Hao, Y. Ding, J. Si, X. Hou, Langmuir 2011, 27, 359; e) S. Wu, D. Wu, J. Yao, Q. Chen, J. Wang, L. Niu, H. Fang, H. Sun, Langmuir 2010, 26, 12012.

[14] a) J. Yong, Q. Yang, F. Chen, D. Zhang, G. Du, H. Bian, J. Si, X. Hou, RSC Adv. 2014, 4, 8138; b) X. Yang, X. Liu, Y. Lu, J. Song, S. Huang, S. Zhou, Z. Jin, W. Xu, J. Phys. Chem. C 2016, 120, 7233; c) A. V. Belyaev, O. I. Vinogradova, Phys. Rev. Lett. 2011, 107, 098301; d) Y. Wan, Z. Lian, J. Xu, Z. Weng, X. Yin, H. Yu, Micro. Nano. Lett. 2014, 9, 712. [15] a) C. Wang, T. Hsueh, J. Phys. Chem. C 2014, 118, 12399; b) E. Mele, S. Girardo, D. Pisignano, Langmuir 2012, 28, 5312; c) J. Yong, Q. Yang, F. Chen, D. Zhang, U. Farooq, G. Du, X. Hou, J. Mater. Chem. A 2014, 2, 5499.

[16] a) J. Y. Chung, J. P. Youngblood, C. M. Stafford, Soft Matter. 2007, 3, 1163; b) S. Zhao, H. Xia, D. Wu, C. Lv, Q. Chen, K. Ariga, L. Liue, H. Sun, Soft Matter. 2013, 9, 4236; c) 


\section{WILEY-VCH}

S. Wu, J. Wang, L. Niu, J. Yao, D. Wu, A. Li, Appl. Phys. Lett. 2011, 98, 081902; d) F. Pirani,

A. Angelini, S. Ricciardi, F. Frascella, E. Descrovi, Appl. Phys. Lett. 2017, 110, 101603.

[17] a) M. Ebara, K. Uto, N. Idota, J. M. Hoffman, T. Aoyagi, Adv. Mater. 2012, 24, 273;

b) J. H. Jang, C. Y. Koh, K. Bertoldi, M. C. Boyce, E. L. Thomas, Nano Lett. 2009, 9, 2113; c)

A. Espinha, M. C. Serrano, Á. Blanco, C. López, Adv. Opt. Mater. 2014, 2, 516; d) Y. Fang, Y. Ni, B. Choi, S. Y. Leo, J. Gao, B. Ge, C. Taylor, V. Basile, P. Jiang, Adv. Mater. 2015, 27, 3696.

[18] a) M. Frensemeier, J. S. Kaiser, C. P. Frick, A. S. Schneider, E. Arzt, R. S. Fertig III, E. Kroner, Adv. Funct. Mater. 2015, 25, 3013; b) J. X. Cui, D. M. Drotlef, I. Larraza, J. P. Fernandez-Blazquez, L. F. Boesel, C. Ohm, M. Mezger, R. Zentel, A. del Campo, Adv. Mater. 2012, 24, 4601; c) D. M. Le, K. Kulangara, A. F. Adler, K. W. Leong, V. S. Ashby, Adv. Mater. 2011, 23, 3278; d) S. Reddy, E. Arzt, A. del Campo, Adv. Mater. 2007, 19, 3833.

[19] a) C. M. Chen, S. Yang, Adv. Mater. 2014, 26, 1283; b) T. Lv, Z. Cheng, D. Zhang, E. Zhang, Q. Zhao, Y. Liu, L. Jiang, ACS Nano. 2016, 10, 9379; c) H. Shahsavan, S. M. Salili, A. Jákli, B. Zhao, Adv. Mater. 2015, 27, 6828; d) W. Wang, J. Salazar, H. Vahabi, A. Joshi-Imre, W. E. Voit, A. K. Kota, Adv. Mater. 2017, 29, 1700295; d) J. Wang, L. Sun, M. Zou, W. Gao, C. Liu, L. Shang, Z. Gu, Y. Zhao, Sci. Adv. 2017, 3 , e1700004; e) T. Lv, Z. Cheng, E. Zhang, H. Kang, Y. Liu, L. Jiang, Small 2017, 13, 1503402.

[20] a) J. S. Leng, X. Lan, Y. J. Liu, S. Y. Du, Prog. Mater. Sci. 2011, 56, 1077; b) J. Hu, Y. Zhu, H. Huang, J. Lu, Prog. Polym. Sci. 2012, 37, 1720; c) M. D. Hager, S. Bode, C. Weber, U. S. Schubert, Prog. Polym. Sci. 2015, 49-50, 3.

[21] T. Xie, Polymer 2011, 52, 4985.

[22] A. B. D. Cassie, S. Baxter, Trans. Faraday Soc. 1944, 40, 546.

[23] J. P. Youngblood, T. J. McCarthy, Macromolecules 1999, 32, 6800.

[24] a) N. A. Patankar Langmuir 2004, 20, 7097; b) N. A. Patankar Langmuir 2004, 20, 8209; c) D. Vollmer, H. J. Butt, Nat. Phys. 2014, 10, 475. 


\section{WILEY-VCH}

[25] a) S. Xing, J. Jiang, T. Pan, Lab Chip. 2013, 13, 1937; b) J. Shiu, C. Kuo, W. Whang, P. Chen, Lab Chip. 2010, 10, 556; c) A. I. Neto, C. R. Correia, C. A. Custódio, J. F. Mano, Adv. Funct. Mater. 2014, 24, 5096.

[26] a) Y. Lai, F. Pan, C. Xu, H. Fuchs, L. Chi, Adv Mater. 2013, 25, 1682; b) C. Wang, T. Hsueh, J. Phys. Chem. C 2014, 118, 12399; c) X. Yang, X. Liu, Y. Lu, J. Song, S. Huang, S. Zhou, Z. Jin, W. Xu, J. Phys. Chem. C 2016, 120, 7233; d) J. Yong, Q. Yang, F. Chen, D. Zhang, U. Farooq, G. Du, X. Hou, J. Mater. Chem. A 2014, 2, 5499.

[27] a) M. Liu, Y. Zheng, J. Zhai, L. Jiang, Acc. Chem. Res. 2010, 43, 368; b) Z. Cheng, M. Du, H. Lai, N. Zhang, K. Sun, Nanoscale 2013, 5, 2776; c) E. Zhang, Y. Wang, T. Lv, L. Li, Z. Cheng, Y. Liu, Nanoscale 2015, 7, 6151. 


\section{WILEY-VCH}

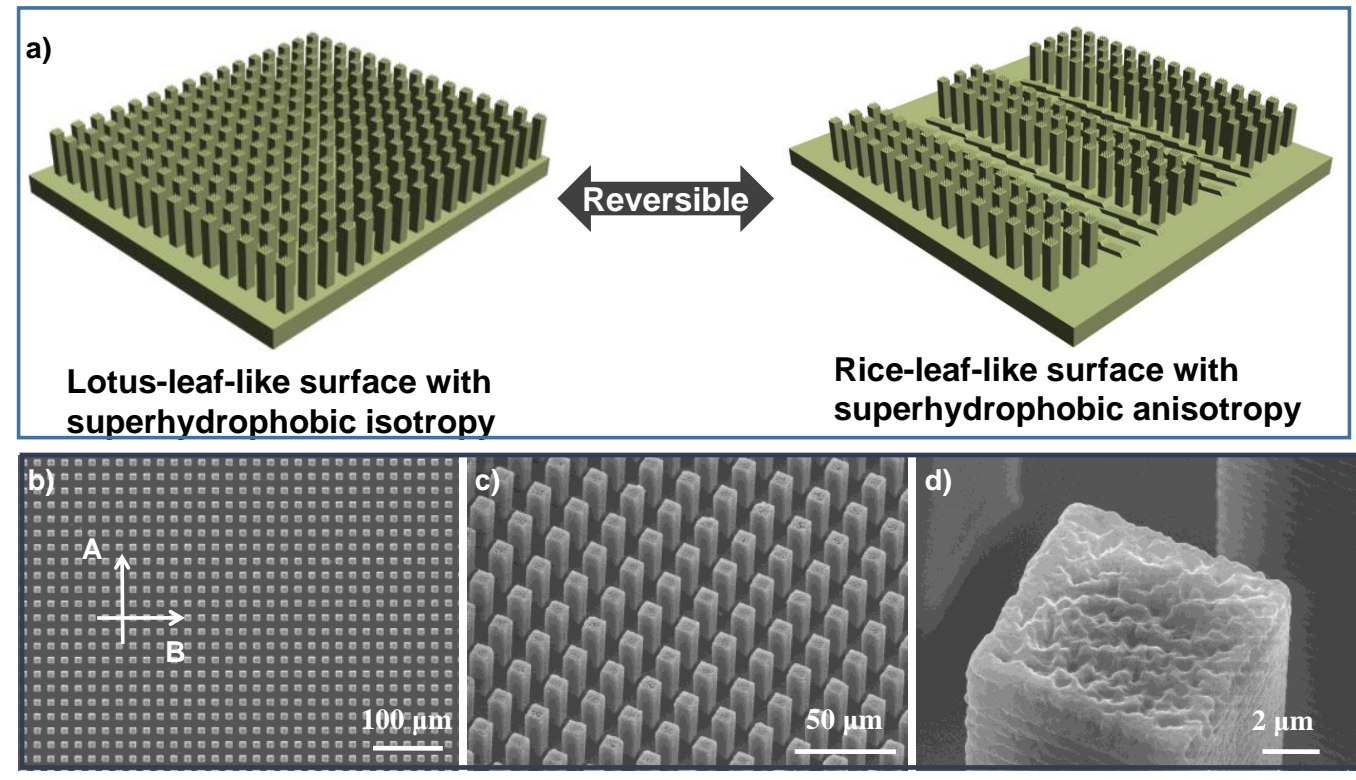

Introducing microgrooves After recovery
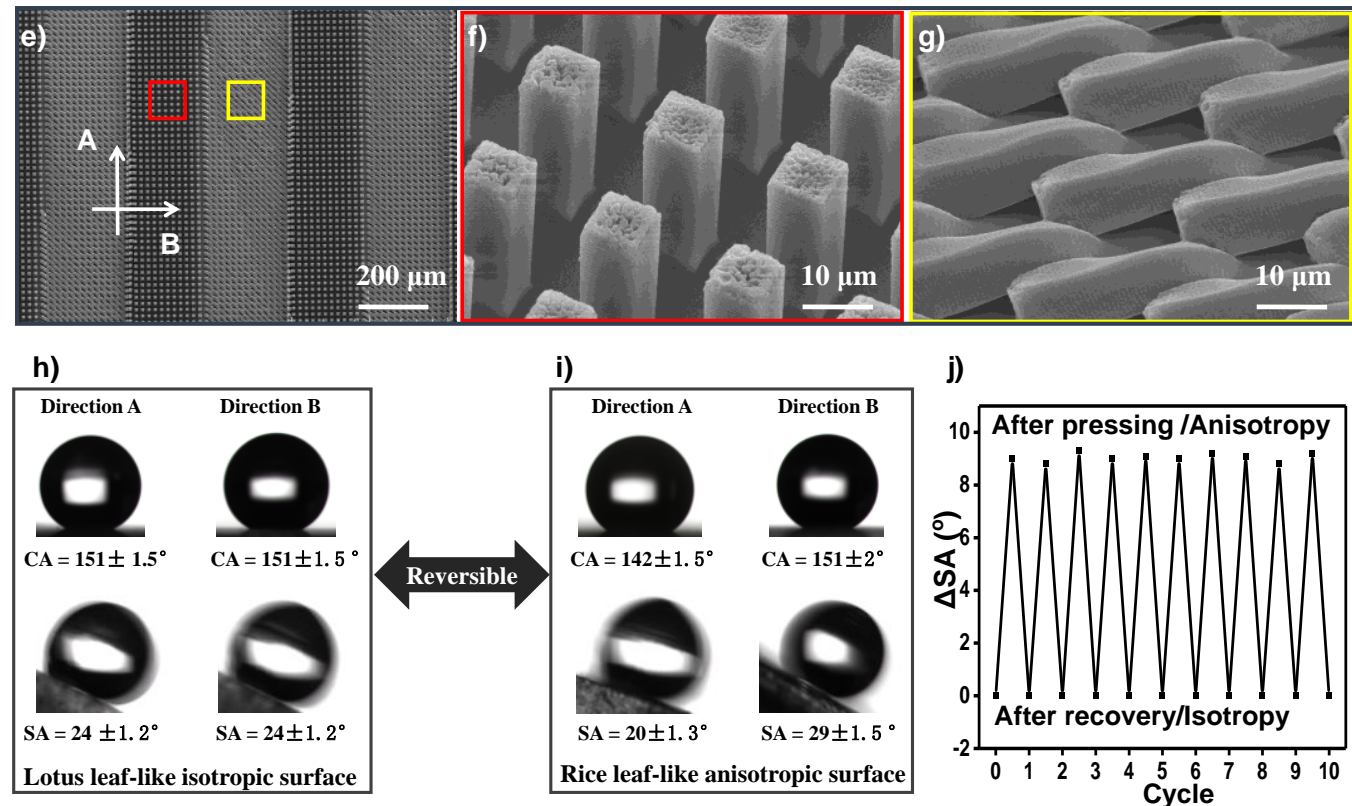

Figure 1. (a) Schematic illustration of the surface microstructure variation between the lotusleaf-like and rice-leaf-like structure shapes. (b) SEM images of the as-prepared/recovered surface. (c, d) amplified images viewed at tilt angle of about $40^{\circ}$ corresponding to (b) at different magnified scales. (e) SEM image of the surface after pressing by a groove structured silicon template with $\mathrm{W}_{\mathrm{g}}$ of about $260 \mu \mathrm{m}$, it can be seen that groove structure can be produced onto the surface after pressing. (f) and (g) are magnified SEM images viewed at tilt angle of about $40^{\circ}$ corresponding to the unchanged (red square) and collapsed (yellow square) pillars, respectively. (h) and (i) are wetting results (CAs and SAs) on the surface corresponding to (b) and (e), respectively. Direction A and B are the measured directions as labeled in image (b) and (e). (j) Variation of difference value for SA $(\Delta \mathrm{SA})$ between the two directions after several consecutive pressing/recovering cycles. From these results, one can find that both static and dynamic wetting performances can be controlled reversibly between the superhydrophobic isotropy and anisotropy on the surface by tuning the surface microstructure. 

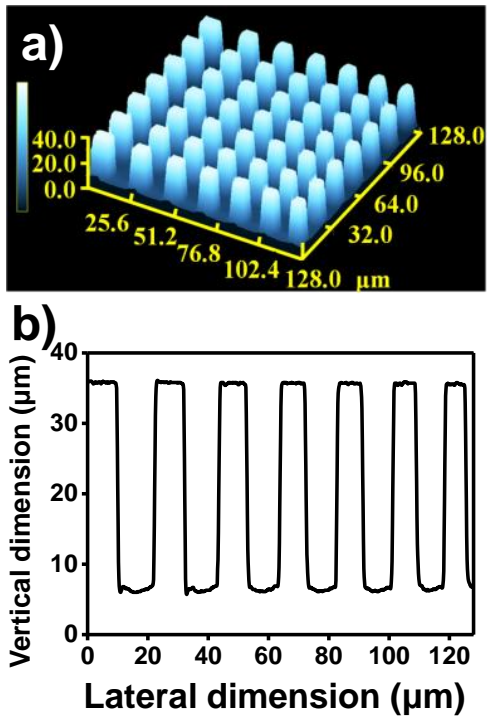

c)

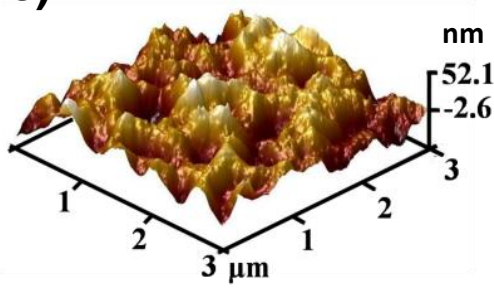

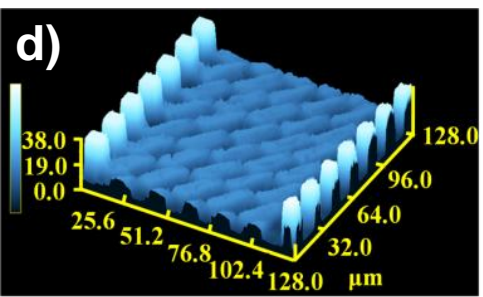

e)

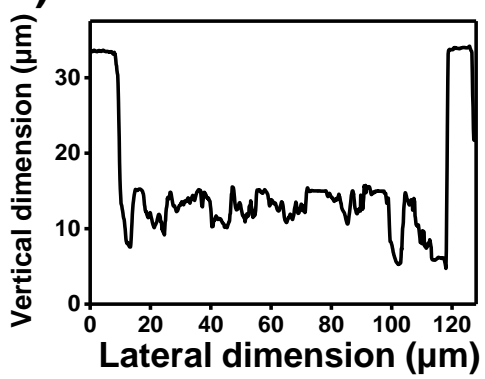

f)

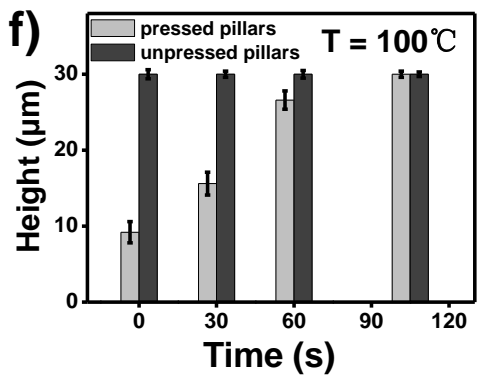

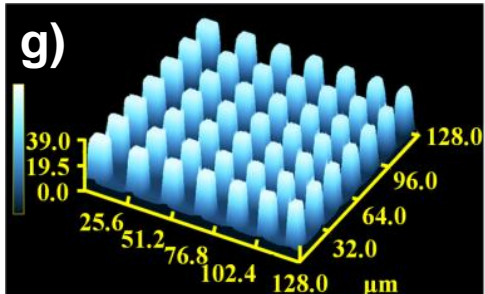

h)
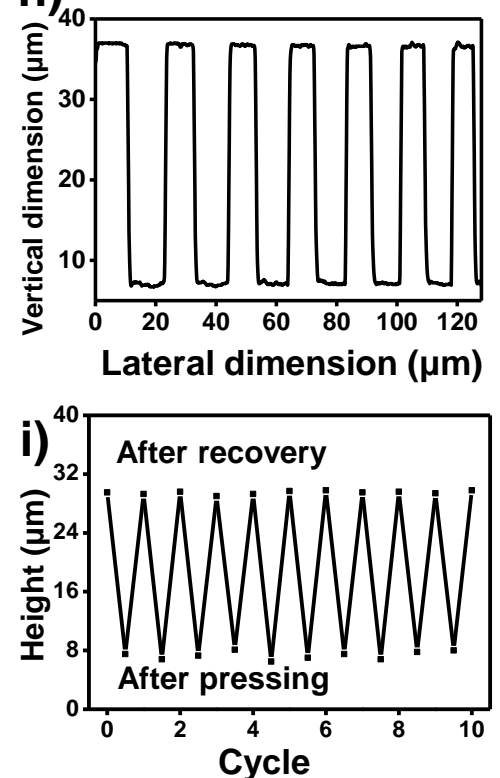

Figure 2. (a), (d), and (g) are the 3D confocal microscopy images for the as-prepared surface, after pressing by a groove structured template, and after recovery, respectively. (b), (e), and (h) are the profile images corresponding to (a), (d), and (g), respectively. (c) AFM image of one pillar tip, confirming the presence of nanostructures. (f) Statistic of average heights of pillars under heating at $100^{\circ} \mathrm{C}$ for different time. (i) Average pillar height for those pressed pillars after several consecutive pressing and recovery cycles. 

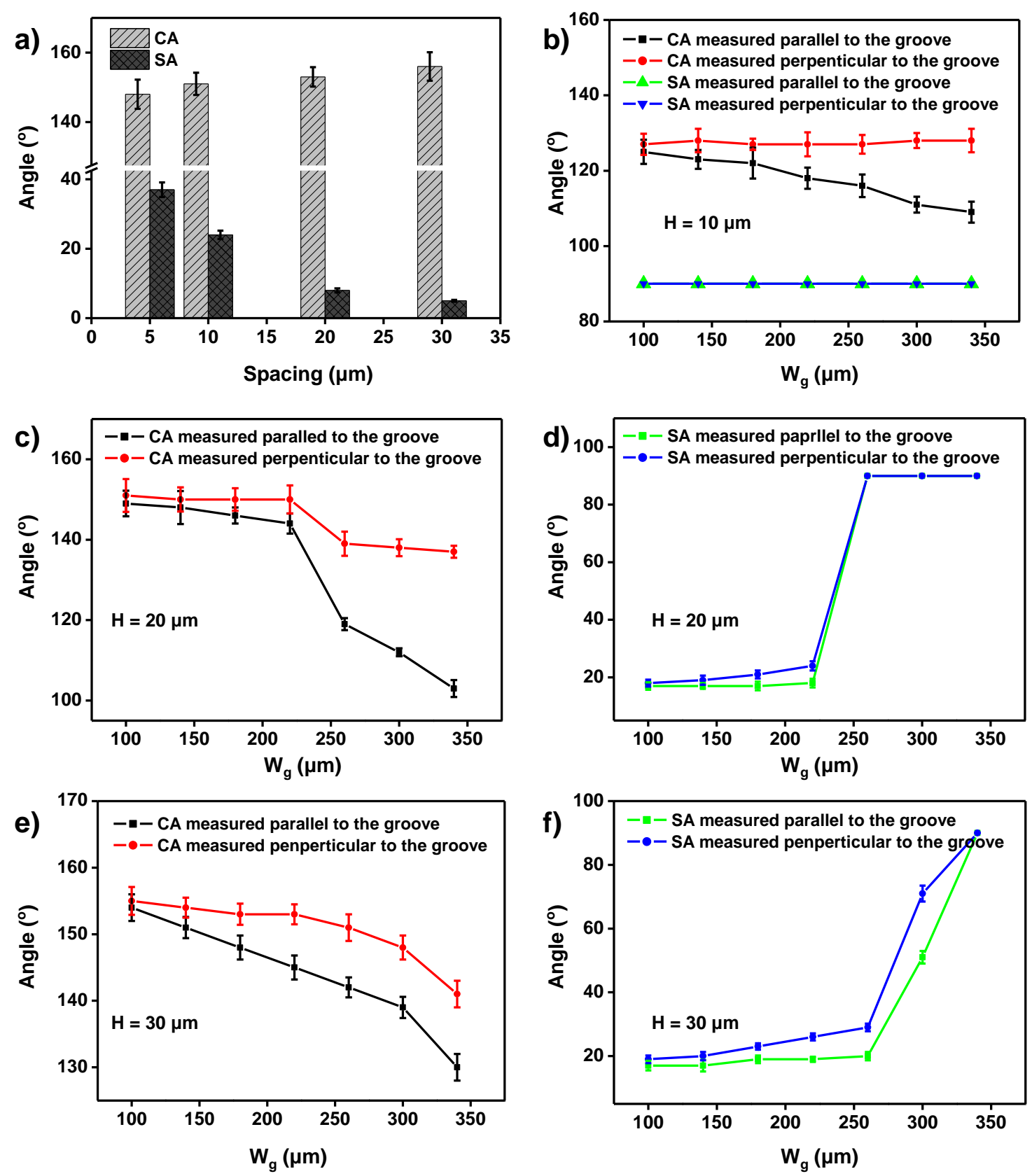

Figure 3. (a) Statistic of CAs and SAs on surfaces with different spacing (all pillars with the same height of $30 \mu \mathrm{m})$. Dependence of CAs/SAs on the $\mathrm{W}_{\mathrm{g}}$ for surfaces with different pillar heights: (b) $\mathrm{H}=10 \mu \mathrm{m}$, (c) and (d) $\mathrm{H}=20 \mu \mathrm{m}$, (e) and (f) $\mathrm{H}=30 \mu \mathrm{m}$, respectively. $\mathrm{SA}=90^{\circ}$ means that droplet is pinned on the surface. 

a)

Heating

1

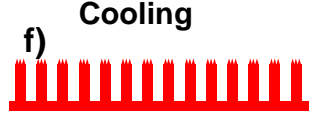

Recovering

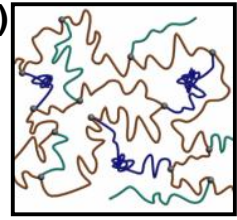

b)

||||||||||||||

h)

Pressure induced

deformation
After pressing

After recovery

e)

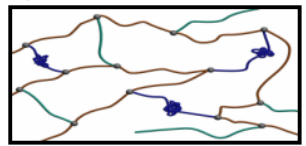

c)

Groove template

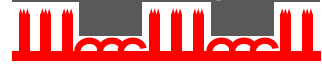

Cooling under

pressure

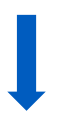

d)

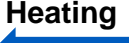

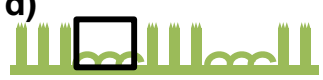

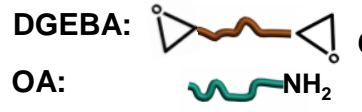
MXDA: $\mathrm{H}_{2} \mathrm{~N} \sim \mathrm{NH}_{2}$ Physical crosslinking:

Chemical crosslinking:

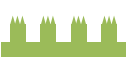
:Molecular chains with low mobility I'I"I"U" :Molecular chains with high mobility
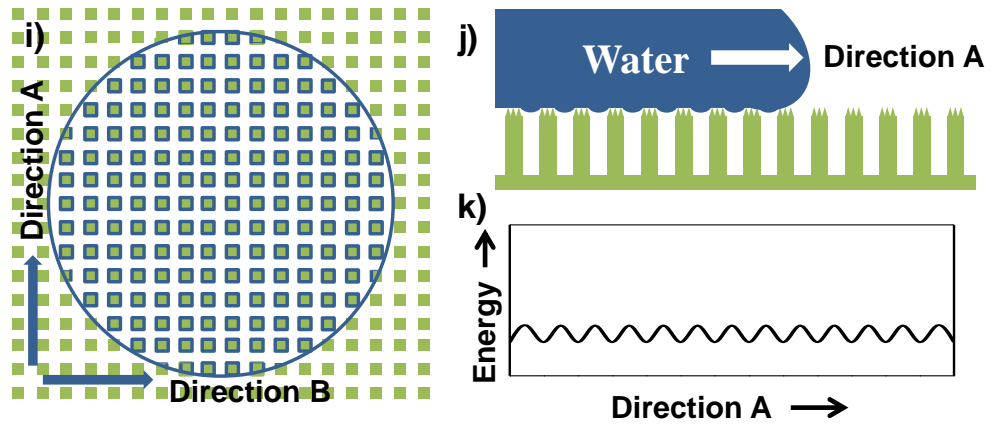

I)
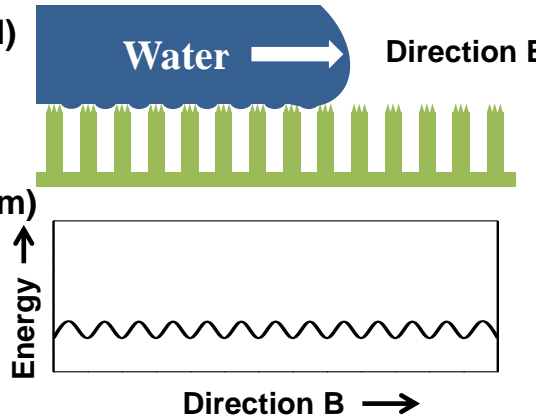

Surface without microgrooves shows superhydrophobic isotropy

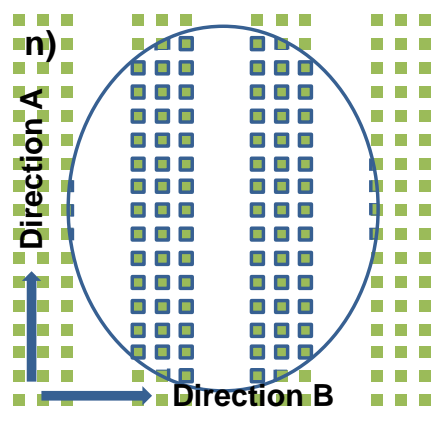

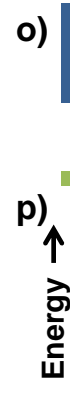

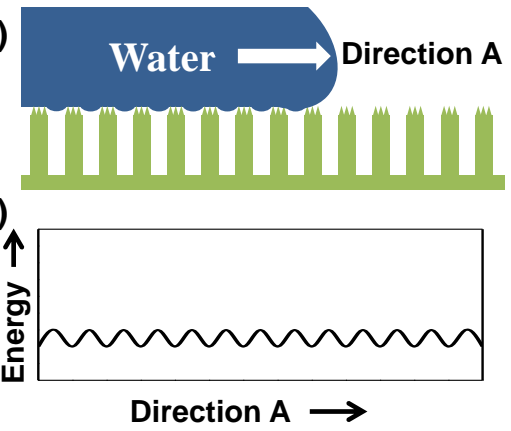

q)

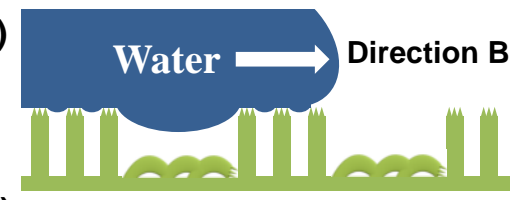

r)

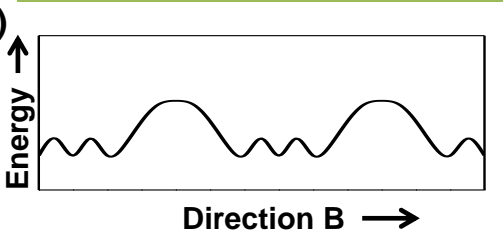

Surface with microgrooves shows superhydrophobic anisotropy

Figure 4. Schematic illustration of variation of the molecular chain conformation, surface microstructure and related solid/liquid contact states. The as-prepared micro/nanostructured pillars are the permanent structure shape (a), which contains lots of crosslinking network and the conformation of the molecular chains has the highest entropy $(\mathrm{g})$. When the surface is heated above $\mathrm{T}_{\mathrm{g}}$ and pressed by a groove-structured template $(\mathrm{b}-\mathrm{c})$, the crosslinking network can prohibit the long-range chain slippage, which can result in the formation of new chain conformation with lower entropy (h) and the collapse of some pillars (c). After cooling, the deformed shape can be kept due to the freeze of the molecular chain segments (d). Further reheating can re-activate the mobility of molecular chains (e) and realize the recovery of chains conformation, the surface structure to the original state due to the thermodynamically favorable tendency for increasing entropy (f). (i) and (n) are schematic illustration of TCL from the top view on surface with and without groove structure, respectively. Direction labeled represents the measured direction. On the surface without groove structure, same contact model $(\mathrm{j}, \mathrm{i})$ and energy barrier $(\mathrm{k}, \mathrm{m})$ during water droplets wetting and rolling in two vertical directions can be observed, Therefore, the surface shows isotropic wetting 


\section{WILEY-VCH}

performances. On the groove structured surface, much larger gaps are formed in direction perpendicular to the grooves $(\mathrm{q})$ than that parallel to the grooves (o), which can offer larger energy barrier $(p, r)$ and result in more discontinuous TCL $(n)$, thus endowing the surface with anisotropic wetting.

a)

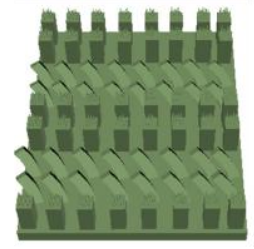

b)

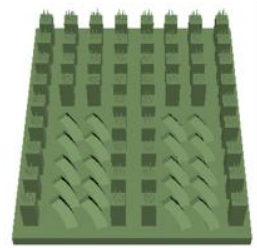

c) Hithestis

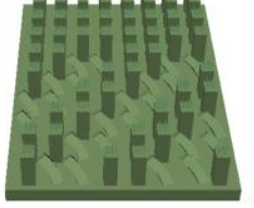

d)

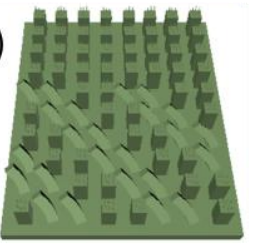

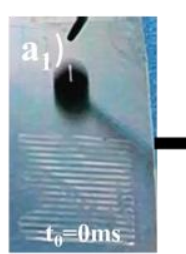
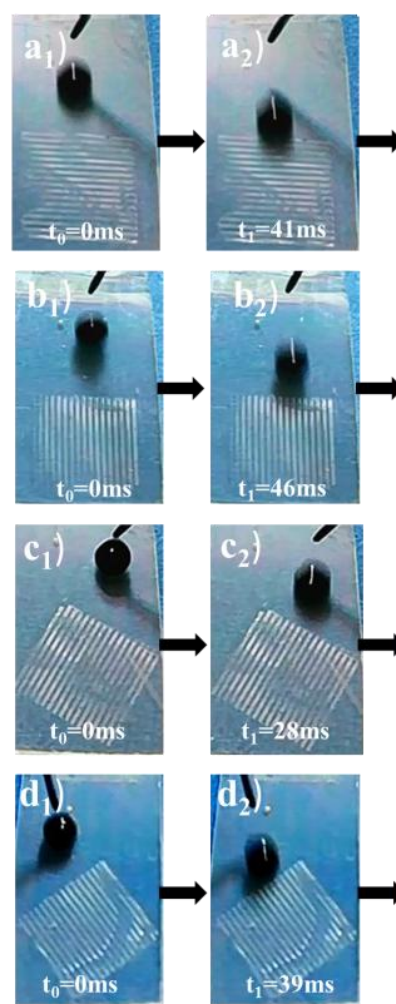
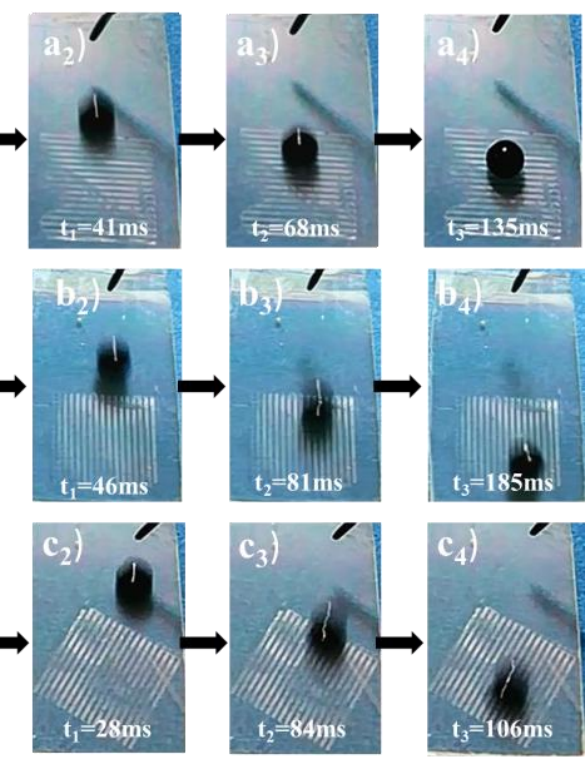

d).

Figure 5. Application of the surface as the rewritable platform for controllable droplet transportation. (a), (b), (c), (d) are the schematic illustration of different designs of surface microstructure on the identical surface, it can be seen that the surface is composed of two parts, the upper part has no groove structure and the bottom part has the groove structure. $\left(a_{1^{-}}\right.$ $\mathrm{d}_{4}$ ) are photographs of the same surface after creating with different microgroove structures through repeatedly pressing/recovering process and corresponding water transportation processes (the color of the droplet is due to the addition of the methylene blue). It can be seen that when the groove structure is in the horizontal direction, the water droplet starts roll from the upper part to the bottom part, when it reaches the region with groove structure, it would be pinned $\left(a_{1}-a_{4}\right)$. When the grooves structures are designed in the vertical direction $\left(b_{1}-b_{4}\right)$, lean to the left direction $\left(c_{1}-c_{4}\right)$, and lean to the right direction $\left(d_{1}-d_{4}\right)$, respectively, the water droplet would roll spontaneously to the corresponding directions as they get to the groove regions. These results indicate that the surface can be used as a rewritable platform and different transportation programmes can be realized. 


\section{WILEY-VCH}

\section{The table of contents entry}

A novel surface that can reversibly transit between the superhydrophobic isotropic and anisotropic wetting states is reported by creating micro/nanostructured pillars on the shape memory polymer. Based on the smart controllability, the surface can be used as the rewritable functional platform, on which various droplet transportation programmes can be redesigned and displayed.

Keyword superhydrophobic, anisotropic/isotropic wetting, shape memory polymer, microstructure, reversible

Zhongjun Cheng,* Dongjie Zhang, Tong Lv, Hua Lai, Enshuang Zhang, Hongjun Kang, Yongzhen Wang, Pengchang Liu, Yuyan Liu,* Yi Du, Shixue Dou and Lei Jiang

Superhydrophobic Shape Memory Polymer Arrays with Switchable Isotropic/Anisotropic Wetting

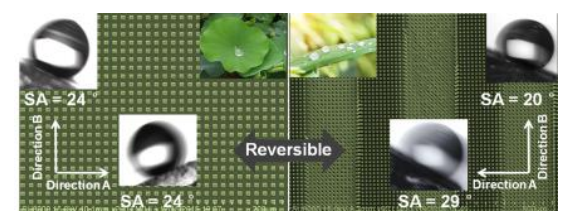

ToC figure ((Please choose one size: $55 \mathrm{~mm}$ broad $\times 50 \mathrm{~mm}$ high or $110 \mathrm{~mm}$ broad $\times 20 \mathrm{~mm}$ high. Please do not use any other dimensions)) 


\section{WILEY-VCH}

((Supporting Information can be included here using this template))

Copyright WILEY-VCH Verlag GmbH \& Co. KGaA, 69469 Weinheim, Germany, 2016.

\section{Supporting Information}

\section{Superhydrophobic Shape Memory Polymer Arrays with Switchable Isotropic/Anisotropic Wetting}

Zhongjun Cheng, * Dongjie Zhang, Tong Lv, Hua Lai, Enshuang Zhang, Hongjun Kang, Yongzhen Wang, Pengchang Liu, Yuyan Liu,* Yi Du, Shixue Dou and Lei Jiang

Table S1. XPS analysis results of flat and microstructured surface under different conditions

\begin{tabular}{cccc}
\hline Sample & \multicolumn{3}{c}{ Atomic concentration(\%) } \\
\cline { 2 - 4 } & $\mathrm{C}$ & $\mathrm{O}$ & $\mathrm{N}$ \\
\cline { 2 - 4 } Flat SMP & 72.54 & 26.05 & 1.41 \\
Microstructure SMP & 73.93 & 24.18 & 1.89 \\
After pressing & 74.45 & 23.51 & 2.04 \\
After recovering & 73.85 & 24.46 & 1.69 \\
\hline
\end{tabular}

From the Table S1, one can observe that the microstructured SMP has the similar chemical composition with that on the flat SMP, meanwhile, during the whole variation of surface microstructure, the surface chemical composition has no apparent variation.

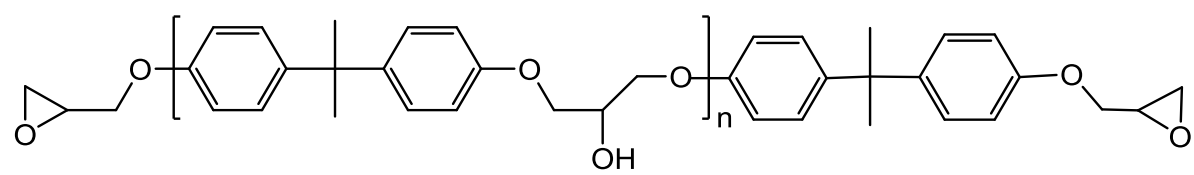

Diglycidyl ether of bisphenol A type epoxy resin E-51(DGEBA)<smiles>CC(C)(C)CC(C)(C)CN</smiles>

n-octylamine $(O A)$<smiles>NCc1cccc(CN)c1</smiles>

m-xylylenediamine (MXDA)

Figure S1. Chemical structures of the SMP prepolymer ingredients. 


\section{WILEY-VCH}

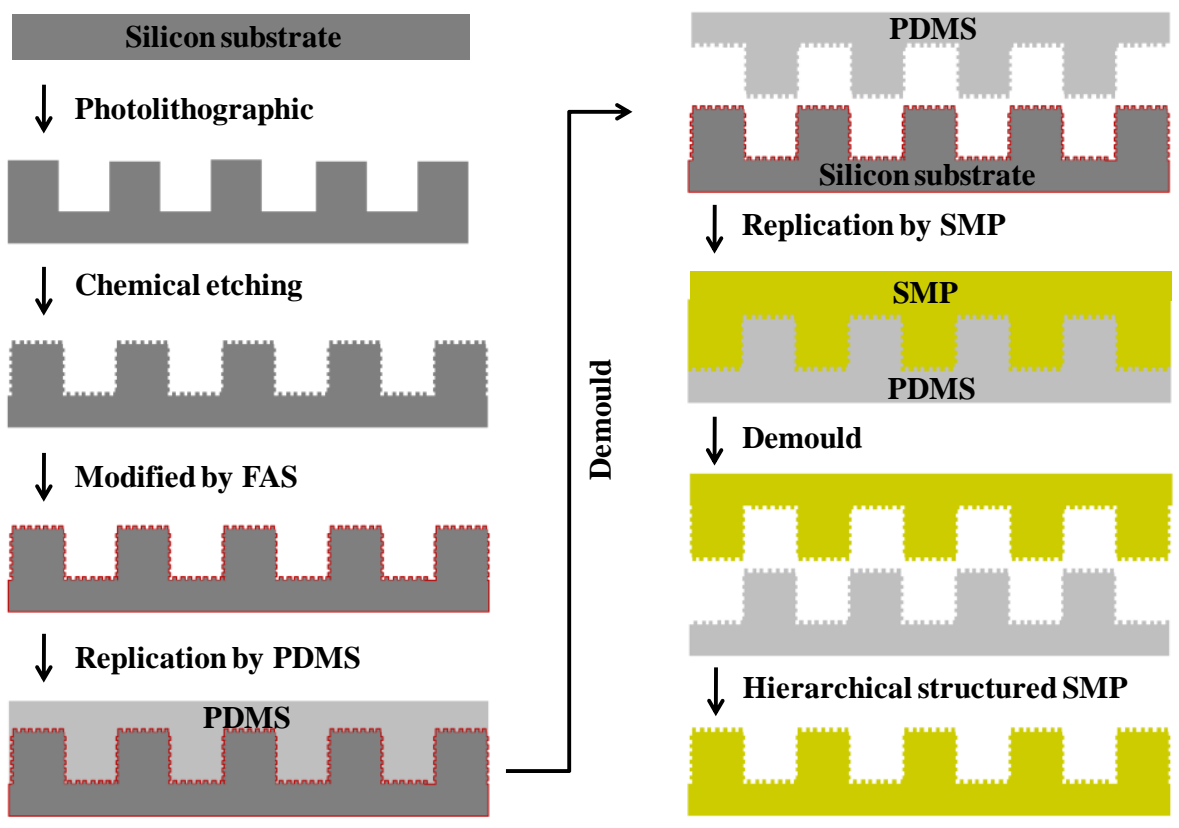

Figure S2. Schematic illustration of preparation process of the lotus-leaf-like hierarchical structured superhydrophobic shape memory polymer surface, the detailed process see experimental section.
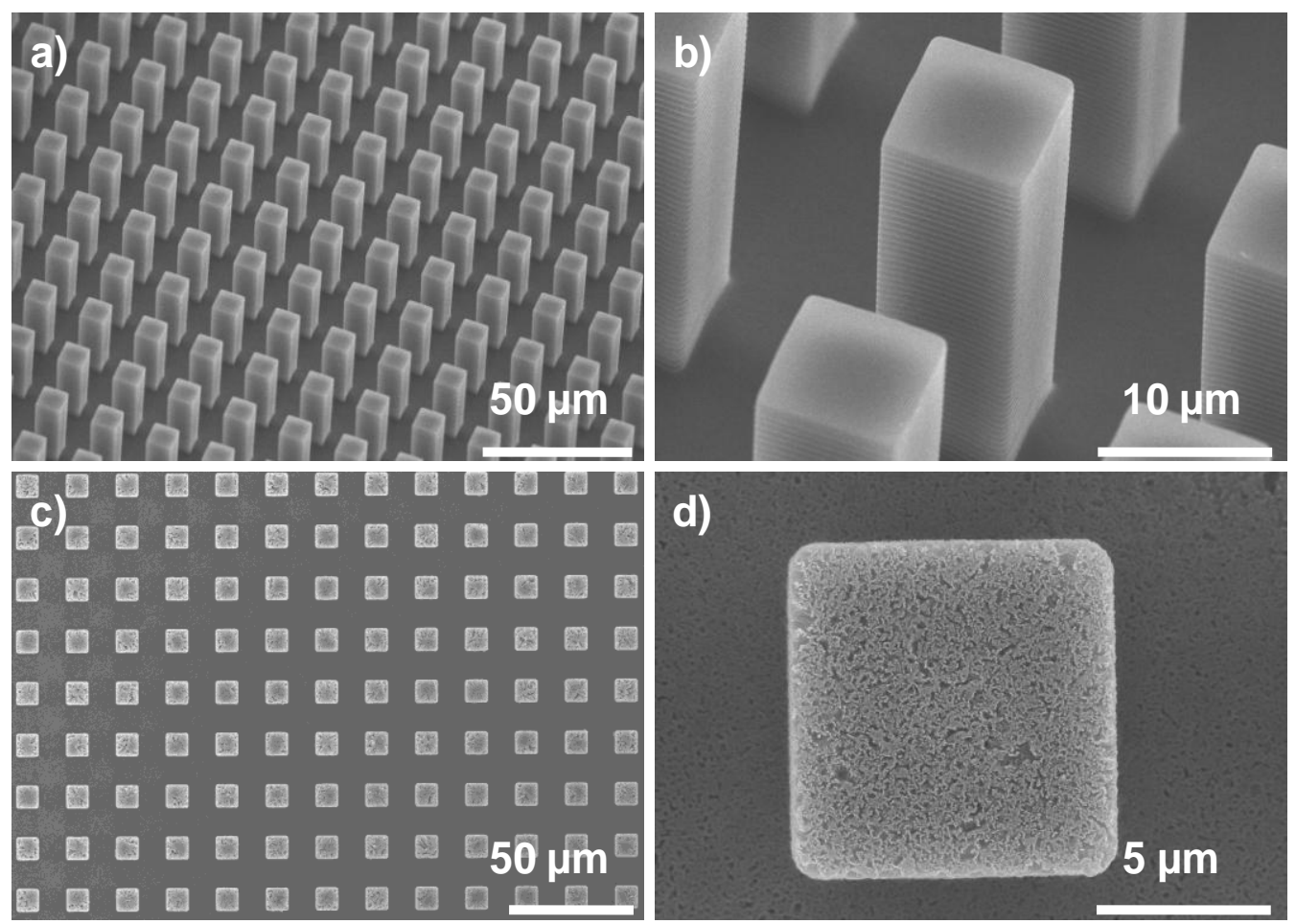

Figure S3. SEM images of the pillar-structured silicon surfaces before (a, b) and after (c, d) chemical etching. (b) and (d) are the magnified images corresponding to (a) and (c), respectively. From these pictures, one can find that after chemical etching, nanostructures can be produced onto the pillar surfaces. 


\section{WILEY-VCH}

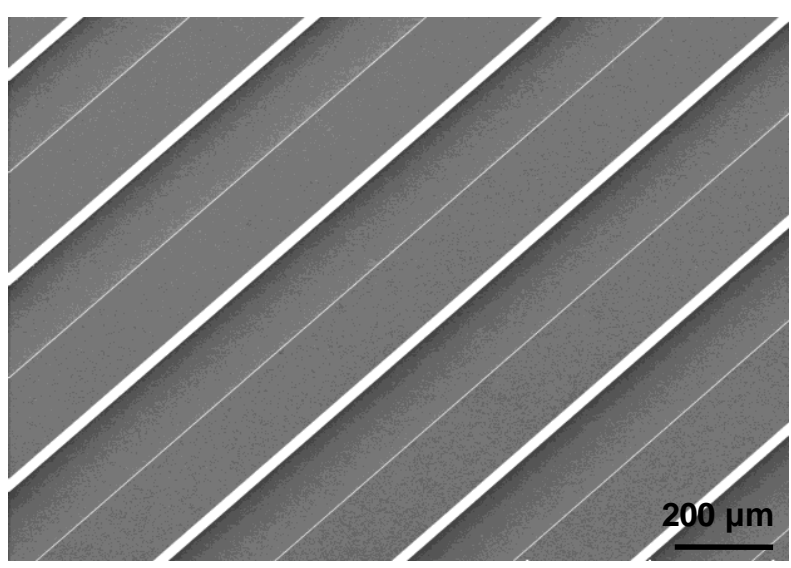

Figure S4. SEM images of the groove structured template with width and depth of $140 \mu \mathrm{m}$ and $50 \mu \mathrm{m}$, respectively. Other templates with different groove widths $(100 \mu \mathrm{m}, 180 \mu \mathrm{m}, 220$ $\mu \mathrm{m}, 260 \mu \mathrm{m}, 300 \mu \mathrm{m}, 340 \mu \mathrm{m})$ and constant depth $(50 \mu \mathrm{m})$ have similar structure.
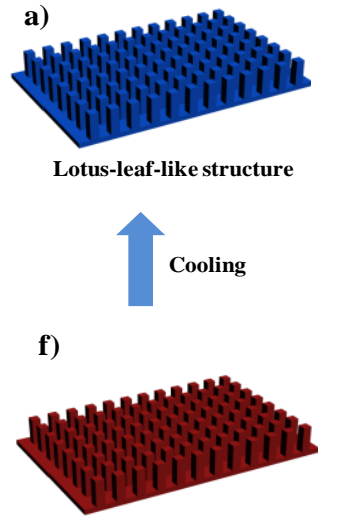
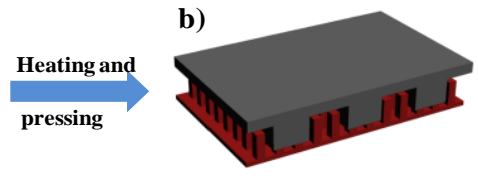
pressing

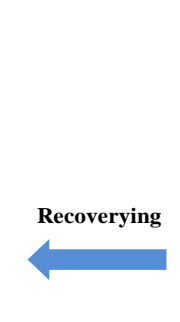

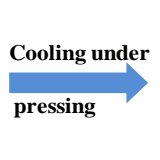

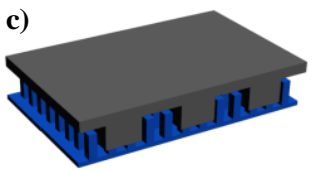

Demolding

d)

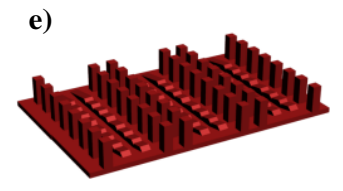

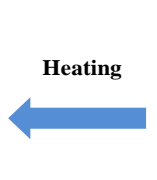

Figure S5. Schematic illustration of the detailed variation process of surface microstructures between the lotus-leaf-like structure and the rice-leaf-like structure.

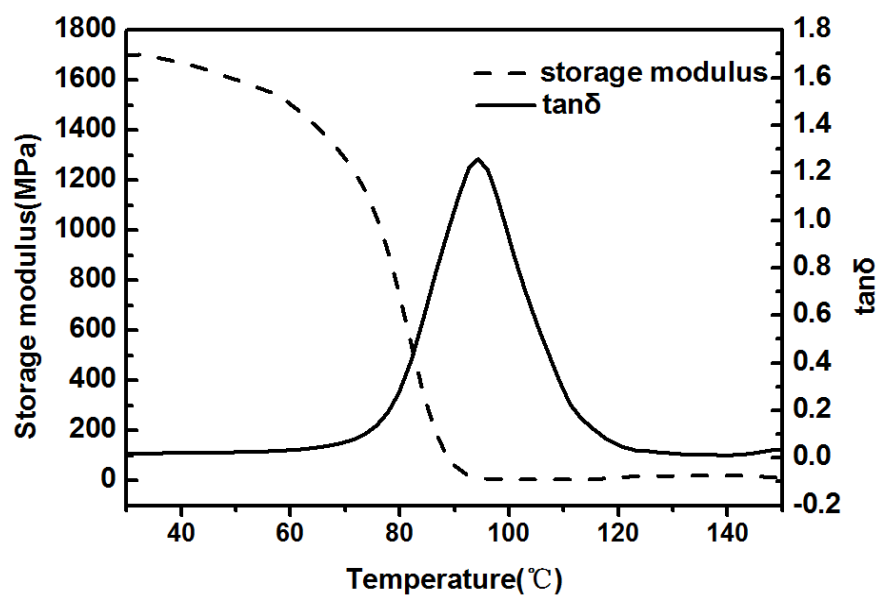

Figure S6. Storage modulus and loss tangent $(\tan \delta)$ as a function of temperature obtained from dynamic mechanical analysis. The $\tan \delta$ peak temperature is $94.3^{\circ} \mathrm{C}$ corresponding to the glass transition temperature $\left(\mathrm{T}_{\mathrm{g}}\right)$ of SMP. The drop of storage modulus with 2.61 order of magnitude indicate that heating can make the materila to become soft. 
WILEY-VCH
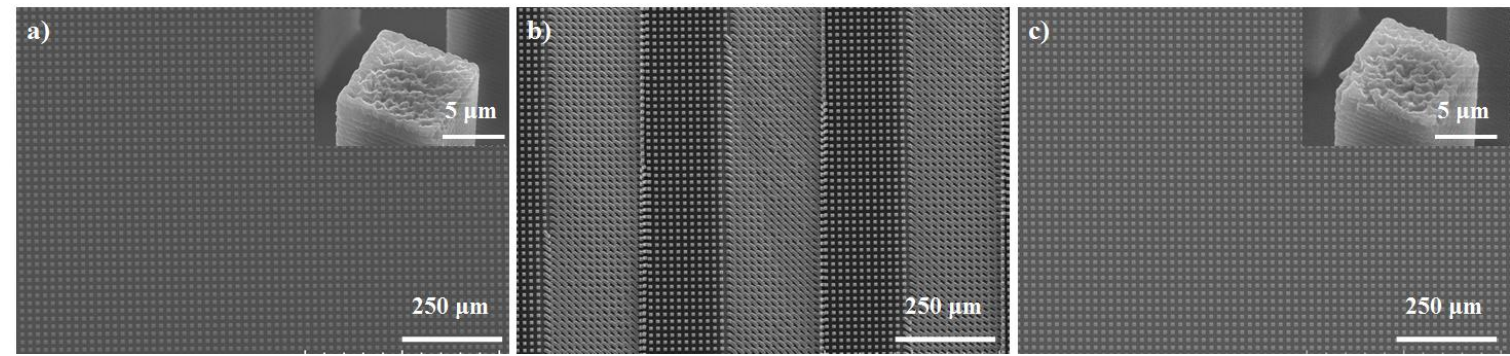

Figure S7. SEM images of the surface before (a), after pressing by a template with groove with width of $260 \mu \mathrm{m}$ (b), and after recovery (c), respectively. Insets are the magnified image of one pillar tip. From these images, it can be seen that surface can memorize different microstructure states, after recovery, the collapsed pillars can recover to the initial state, and nanostructures can still be observed on pillar tip.

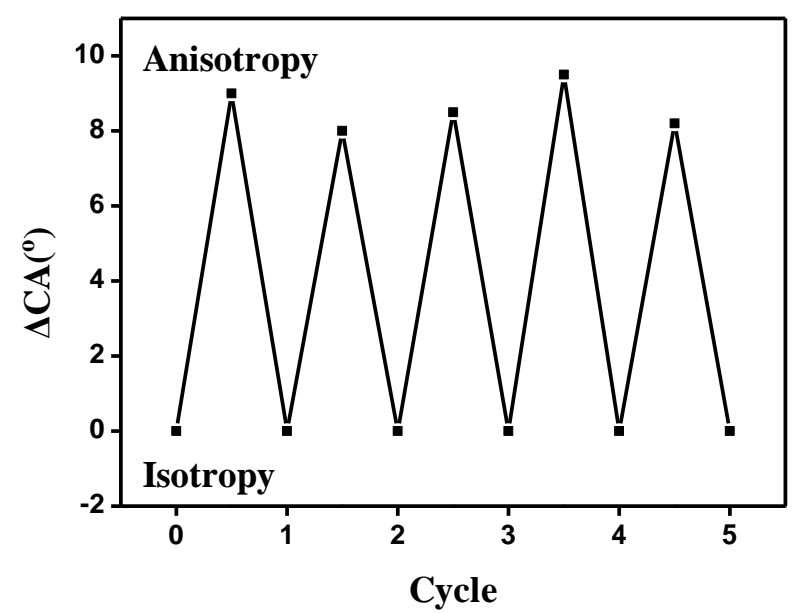

Figure S8 Reversible transition between isotropy and anisotropy characterized by the difference value of static contact angles in different directions (measured perpendicular and parallel to the grooves).

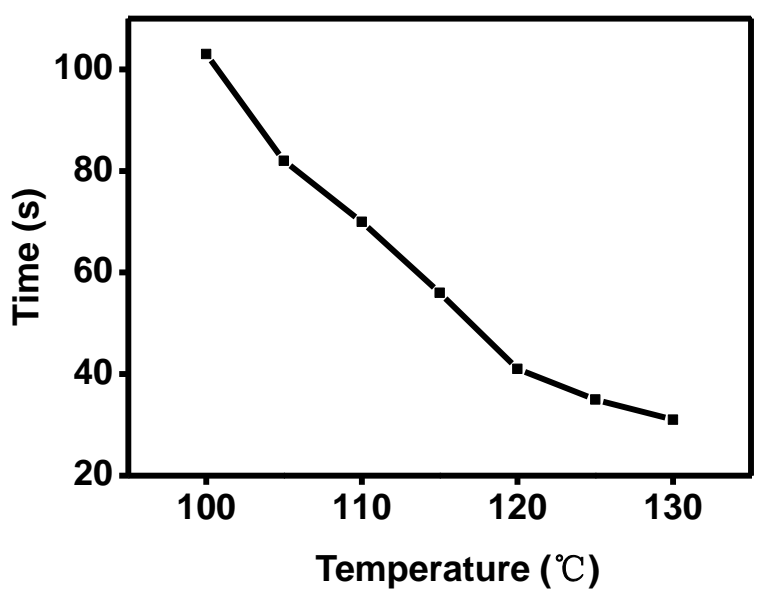

Figure S9. Dependence of needed time on temperature for the recovery of collapsed pillars. It can be seen that as the heating temperature is increased, the needed time is decreased. 


\section{WILEY-VCH}

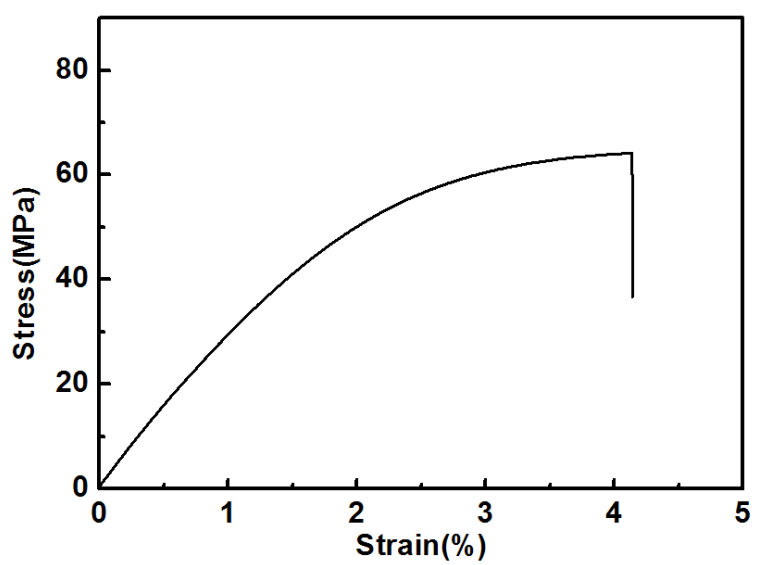

Figure S10. Stress-strain curves of SMP. It can be seen that SMP has a high elongation $(4.14 \%)$ at break, demonstrating a good toughness.

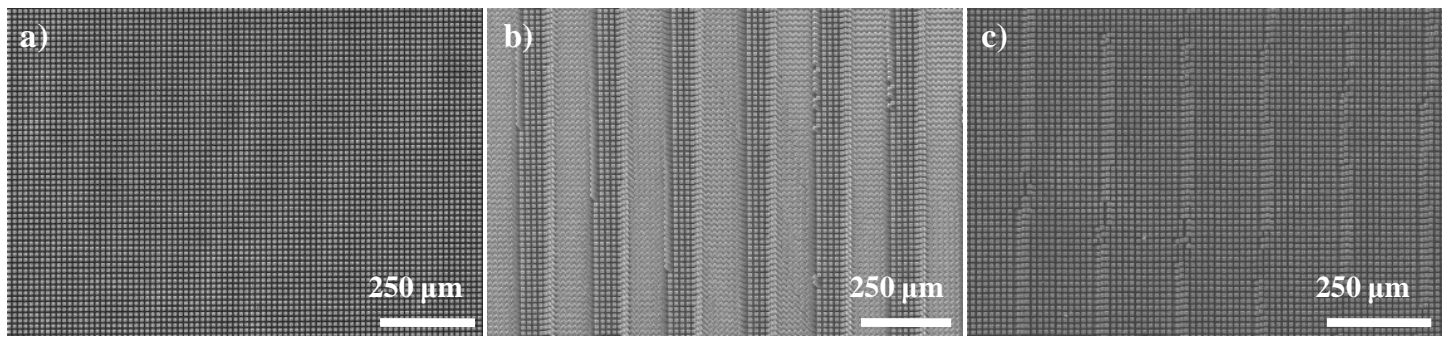

Figure S11. SEM images of the surface with spacing of $5 \mu \mathrm{m}$ before (a), after pressing by the groove structured template (b), and after reheating (c). It can be found that on such a surface, some pillars cannot recover to the original state after reheating because the pillars can be adhered together.
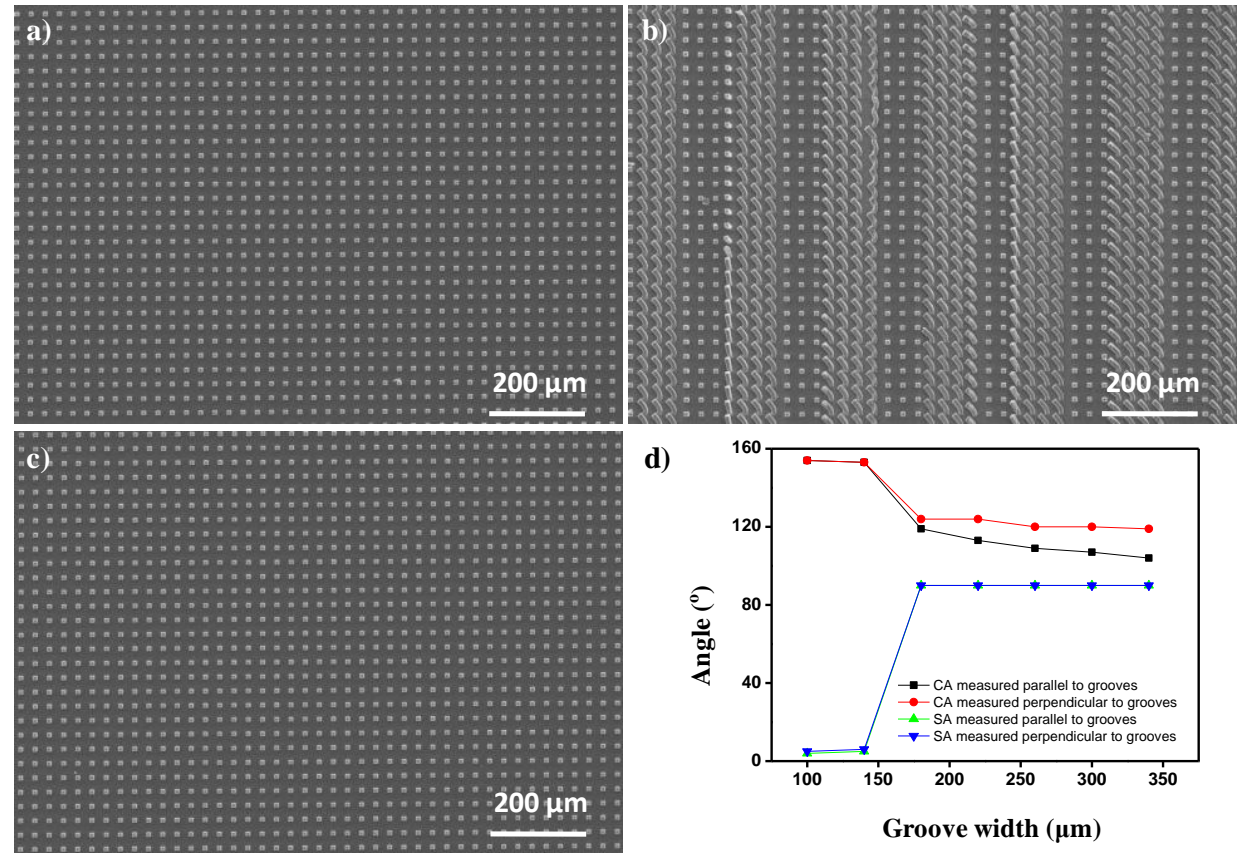

d)

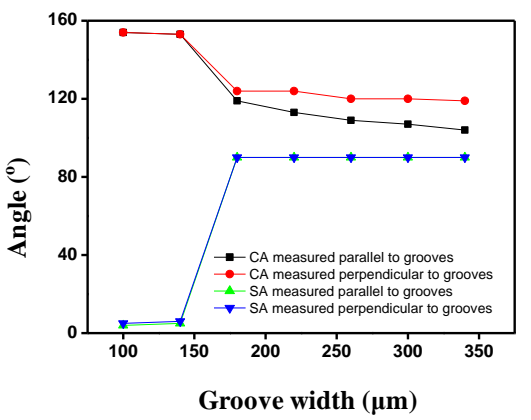

Figure S12. SEM images of the surface with spacing of $20 \mu \mathrm{m}$ before (a), after pressing by the groove structured template (b, groove width is about $100 \mu \mathrm{m})$, and after reheating (c), 


\section{WILEY-VCH}

respectively. What needs to be stressed is that surface with groove width of $100 \mu \mathrm{m}$ just represents one example, surfaces with other groove widths have the similar controllability of the microstructures. (d) Dependence of water contact angles and sliding angles on the width of grooves. It can be seen that, on such a surface, microstructure can be controlled at different states, however, when the microgrooves were introduced onto the surface, the surface cannot display anisotropic wetting (as the groove width is equal or less than $140 \mu \mathrm{m}$ ) or lose the superhydrophobicity (as the groove width is larger than $140 \mu \mathrm{m}$ ).
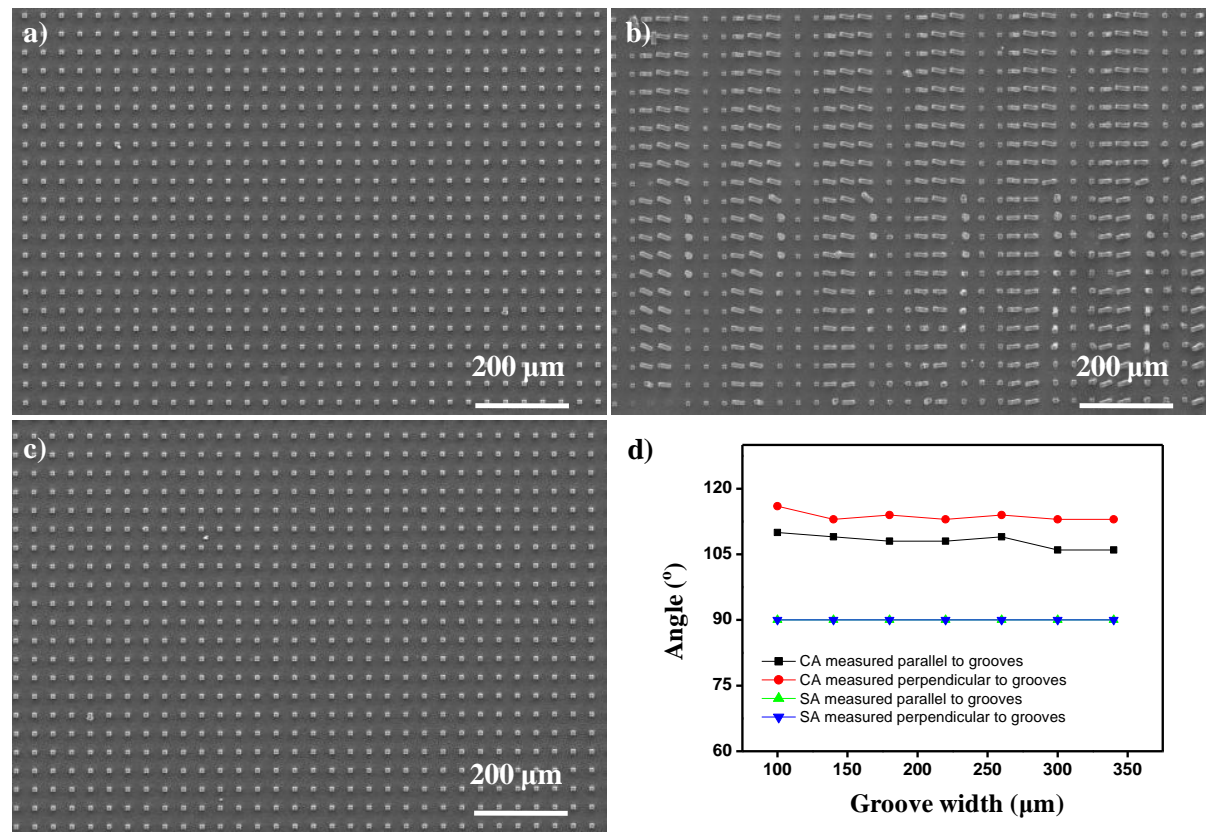

Figure S13. SEM images of the surface with spacing of $30 \mu \mathrm{m}$ before (a), after pressing by the groove structured template (b, groove width is about $100 \mu \mathrm{m})$, and after reheating (c), respectively. What needs to be stressed is that surface with groove width of $100 \mu \mathrm{m}$ just represents one example, surfaces with other groove widths have the similar controllability of the microstructures. (d) Dependence of water contact angles and sliding angles on the width of grooves. It can be seen that, on such a surface, microstructure can be controlled at different states, however, when the microgrooves were introduced onto the surface, the surface would lose the superhydrophobicity and only static anisotropy can be observed. 


\section{WILEY-VCH}

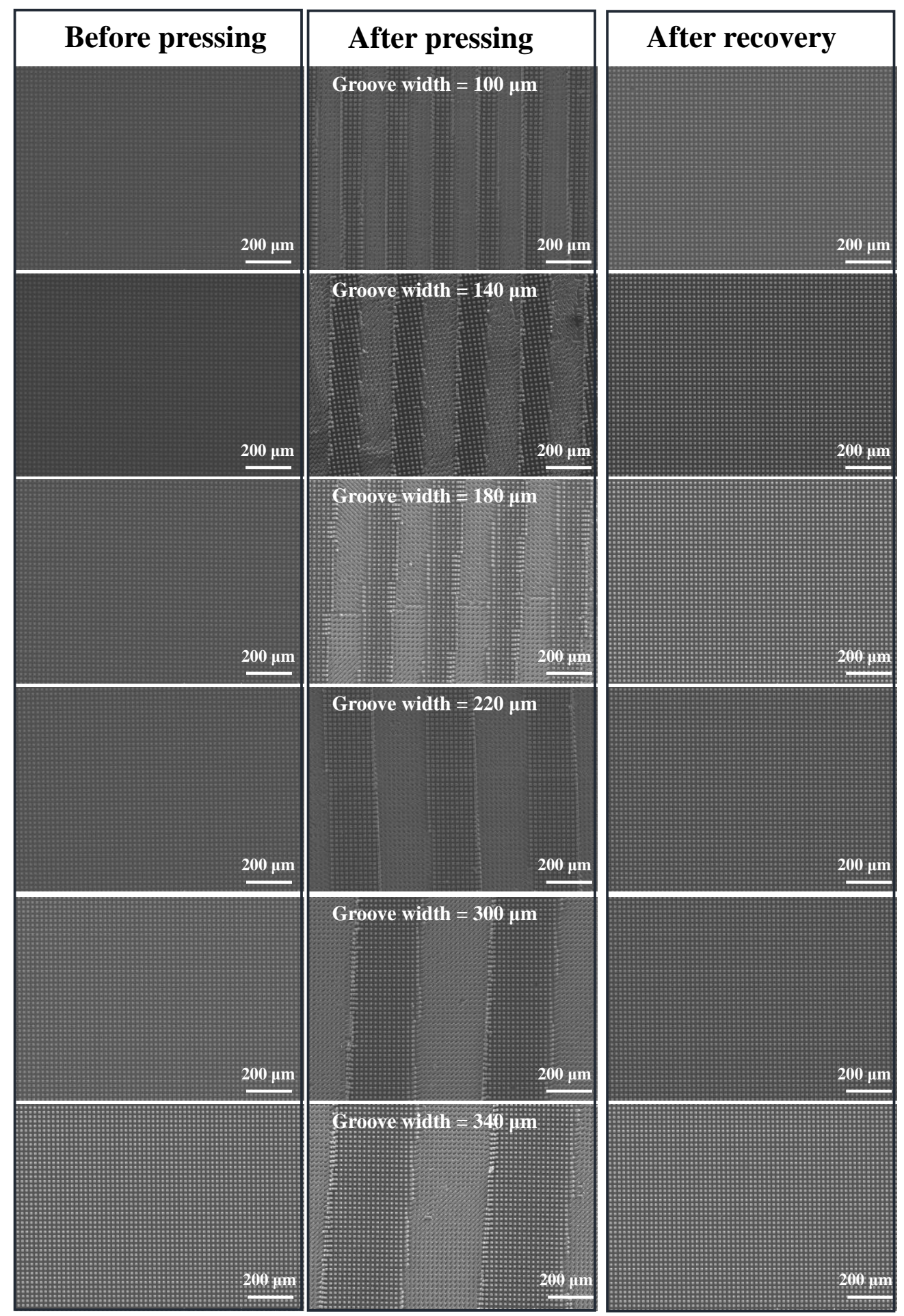

Figure S14. SEM images of the as-prepared surfaces (arrays with pillar spacing and height of $10 \mu \mathrm{m}$ and $30 \mu \mathrm{m}$, respectively) before pressing by the microgroove structured templets (the left column); after pressing by the templets with different groove width (the middle column); and after recovery (the right column). From these images, it can be seen that by adjusting the groove width of the template, surfaces with various grooves widths can be obtained. Meanwhile, all these surface show excellent shape memory effect. For pillars have height of $10 \mu \mathrm{m}$ and $20 \mu \mathrm{m}$, similar phenomena can be observed. 


\section{WILEY-VCH}

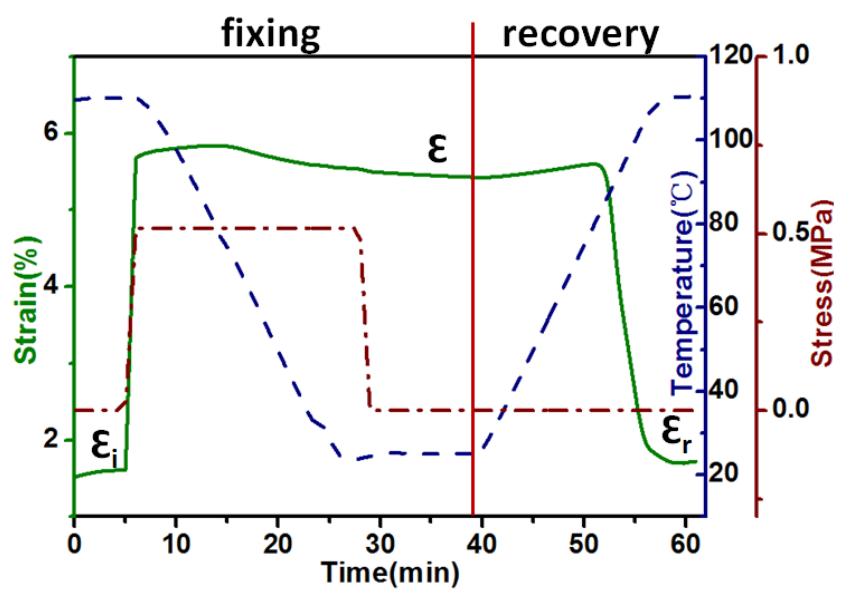

Figure S15. Shape memory quantitative cycle for SMP. Shape recovery ratio $=\left(\varepsilon-\varepsilon_{\mathrm{r}}\right) /(\varepsilon-$ $\left.\varepsilon_{\mathrm{i}}\right) \times 100 \%$. Form the figure, it can be calculated that the shape recovery ratio of the SMP material is $98 \%$.

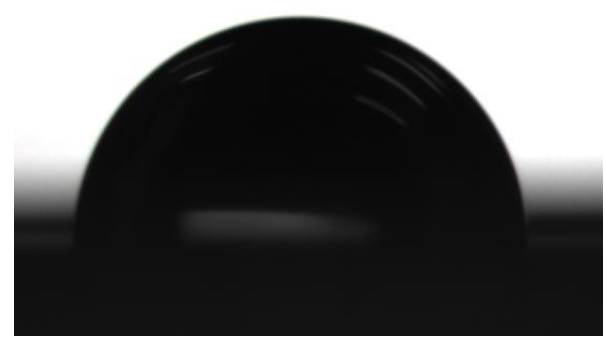

Figure S16. Shape of a water droplet on the flat SMP surface with a contact angle of about $84^{\circ}$.

\section{Discussion about the superhydrophobicity on the surface.}

To have a better understanding about the surface superhydrophobicity, the flat SMP surface with the same chemical composition was also prepared and investigated. As shown in Figure $\mathrm{S} 16$, the contact angle is about $84^{\circ}$ on the flat surface. In this work, as shown in Figure $3 \mathrm{a}$, all surfaces prepared with different spacings $(5 \mu \mathrm{m}, 10 \mu \mathrm{m}, 20 \mu \mathrm{m}, 30 \mu \mathrm{m})$ have the superhydrophobicity, and such high hydrophobicity can be explained by the following equation: ${ }^{[1]}$

$\cos \theta_{c}=f_{1} \cos \theta-f_{2}$

Here $(\theta)$ and $\left(\theta_{c}\right)$ are the contact angles of the flat SMP surface and the rough SMP surface, respectively, $f_{1}$ and $f_{2}$ are the fractions of the solid surface and air in contact with water, respectively (i.e., $f_{1}+f_{2}=1$ ). It is easy to deduce from equation 1 that increasing $f_{2}$ increases $\theta_{c}$, that is, the fraction of air in the surface is an important factor in determining the 


\section{WILEY-VCH}

superhydrophobicity of the surface. Herein, $\theta$ are $84^{\circ}$. For surfaces spacing of $5 \mu \mathrm{m}, 10 \mu \mathrm{m}$, $20 \mu \mathrm{m}, 30 \mu \mathrm{m}$, respectively, $\theta_{c}$ are $151^{\circ}, 152^{\circ}, 154^{\circ}$, and $156^{\circ}$, respectively. According to the above equation, $f_{2}$ are $0.887,0.894,0.909$, and 0.922 , respectively, indicating that as the spacing is increased, more air can be trapped under the droplet, thus, the as shown in Figure 3a, the contact angle is increased, and the present air is high enough to induce the high hydrophobicity and even superhydrophobicity. Meanwhile, because $f_{2}$ is increased as the spacing is increased, accordingly, $f_{1}$ is decreased as the spacing is increased, means that less solid/liquid contact area is formed as the spacing is increased. Therefore, as the spacing is increased, the sliding angle for droplet is decreased.
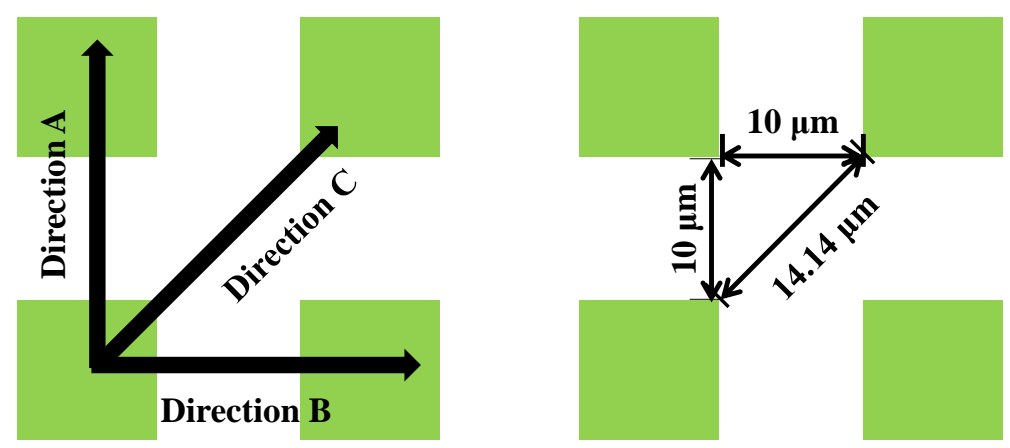

Figure S17. Schematic illustration of the arrangement of the micro/nanostructured pillars.

In our work, we find that on the as-prepared surface, superhydrophobic isotropic wettability can be observed. The reason can be explained as follows. It is well known that the energy barrier is related to the width of the gaps between the pillars. ${ }^{[2]}$ On the as-prepared surface, as shown in Figure S17, the smallest gap is in the direction A and B, and the largest gap is in direction $\mathrm{C}$. In our work, it can be seen that in direction $\mathrm{A}$ and $\mathrm{B}$, the width of the gaps are the same, which are $10 \mu \mathrm{m}$. In direction $\mathrm{C}$, the width is about $14.14 \mu \mathrm{m}$. One can find that the difference between the largest gap and the smallest gap is only about $4.14 \mu \mathrm{m}$. We believe that this difference is too small to cause effective energy barrier difference and corresponding anisotropic wettability in different directions, especially on surface with micro/nanostructures ${ }^{[3]}$ Furthermore, our experimental results can also support it, as shown in 


\section{WILEY-VCH}

Figure $3 \mathrm{e}$ and $3 \mathrm{f}$, even after introduction of groove structures with $\mathrm{W}_{\mathrm{g}}=100 \mu \mathrm{m}$, the anisotropy is still inconspicuous.

a)

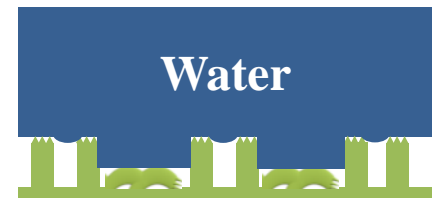

Short pillars cannot impede water wetting grooves

c)

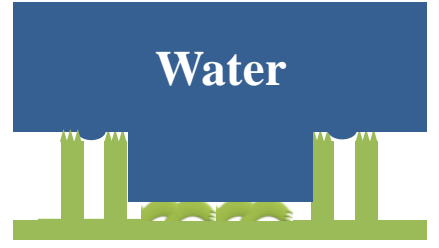

Large $W_{g}$ results in the wetting of grooves b)

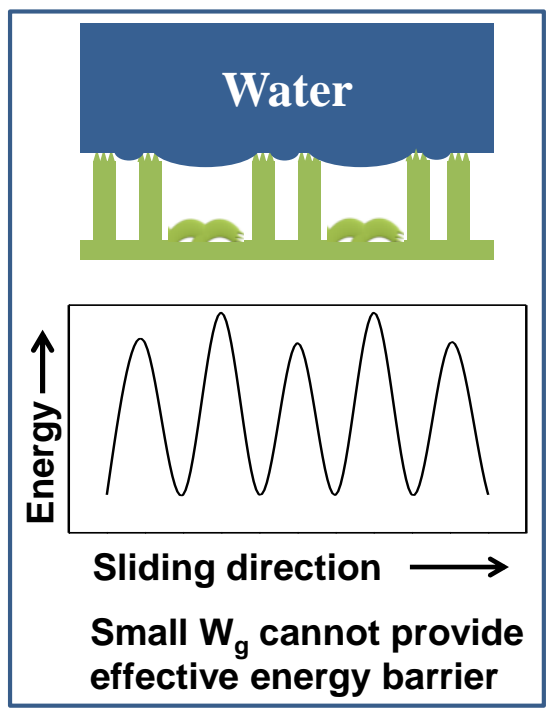

Figure S18. Schematic illustration of solid/water contact states: (a) for surfaces with short pillars, (b) high pillars with small $\mathrm{W}_{\mathrm{g}}$, (c) high pillars large $\mathrm{W}_{\mathrm{g}}$. Results indicate that short pillars and too large $\mathrm{W}_{\mathrm{g}}$ would result in the loss of surface superhydrophobicity, and too small $\mathrm{W}_{\mathrm{g}}$ cannot provide effective energy barrier.

Discussion about the dependence of the wetting performance on the groove width and pillar height on the surfaces.

In this work, before pressing with groove structured template, on all as-prepared surfaces, water droplet can roll with contact angles higher than $150^{\circ}$, means that all these surfaces have the superhydrophobicity and the droplets reside in the composite Cassie state. ${ }^{[1]}$ After introduction of microgroove structures, the wetting performances would be changed. Figure $3 \mathrm{~b}$ shows the variation of water contact angles and sliding angles as a function of groove width on $10 \mu \mathrm{m}$ height pillars. It can be seen that after introduction of microgrooves, the surface would lose the superhydrophobicity. This variation can be explained as follows: according to previous reports, ${ }^{[4-7]}$ increasing the spacing would result in the decrease of the upward Laplace force, and for surface with large distance between pillar, high pillars are needed to obtain the superhydrophobic composite state. In this work, the introduction with 


\section{WILEY-VCH}

microgrooves can result big gaps between the pillars, as a result, the pillar height would be too small to withstand the droplet (Figure S18a), thus water would enter into the gaps, and the surface shows general hydrophobicity. During the wetting process, large solid/liquid contact area can be formed, and therefore, droplets cannot roll on the surface even along the microgrooves. As mentioned above, high pillars are needed for large gaps to obtain the superhydrophobicity. Therefore, as shown in Figure 3c-f, when the pillar height is increased, it can be found that for relative small-width grooves (the widths of the grooves less than 220 $\mu \mathrm{m}$ and $260 \mu \mathrm{m}$ for pillars with height of $20 \mu \mathrm{m}$ and $30 \mu \mathrm{m}$, respectively. Figure $3 \mathrm{c}$ and 3e) the surface can keep the superhydrophobicity and water droplet can still roll on the surface. While when the large-width grooves were introduced, similar with those surfaces with pillar height of $10 \mu \mathrm{m}$, the surface would lose the superhydrophobicity and droplet would be pinned (Figure S18c). These results indicate that for surfaces with different widths of microgrooves, suitable pillar height is needed for the superhydrophobicity. In addition to pillar height, a suitable width of groove is also very important for the anisotropic wetting. It is these grooves that provide the energy barrier and result in the anisotropic wetting. As shown in Figure S18b, when the width of the grooves is too small, there is no apparent difference for energy barriers caused by these grooves compared with that caused by small valleys between pillars. In other words, the energy barriers that water droplets need to overcome are approximate in both directions parallel (the energy barrier resulted from small valley between pillars) and perpendicular (the energy barrier resulted from small valley between pillars and grooves) to the grooves. Thus, as shown in Figure $3 d$ and $3 f$, for surface with small width of groove, the anisotropic wetting performance is not obvious, and when the width of groove is increased, the degree of anisotropy is increased.

\section{References}

[1] A. B. D. Cassie and S. Baxter, Trans. Faraday Soc. 1944, 40, 546. 


\section{WILEY-VCH}

[2] J. P. Youngblood, T. J. McCarthy, Macromolecules 1999, 32, 6800.

[3] X. Gao, Y. Yao, L. Jiang, Langmuir 2007, 23, 4886.

[4] N. A. Patankar Langmuir 2004, 20, 7097.

[5] N. A. Patankar Langmuir 2004, 20, 8209.

[6] Z. Yoshimitsu, A. Nakajima, T. Watanabe, K. Hashimoto, Langmuir 2002, 18, 5818.

[7] D. Vollmer, H. J. Butt, Nat. Phys. 2014, 10, 475. 